\title{
Implications of chiral symmetry on $S$-wave pionic resonances and the scalar charmed mesons
}

\author{
Meng-Lin Du, ${ }^{1, *}$ Feng-Kun Guo, ${ }^{2,3, \dagger}$ and Ulf-G. Meißner ${ }^{1,4,5, \$}$ \\ ${ }^{1}$ Helmholtz-Institut für Strahlen- und Kernphysik and Bethe Center for Theoretical Physics, \\ Universität Bonn, D-53115 Bonn, Germany \\ ${ }^{2}$ CAS Key Laboratory of Theoretical Physics, Institute of Theoretical Physics, \\ Chinese Academy of Sciences, Beijing 100190, China \\ ${ }^{3}$ School of Physical Sciences, University of Chinese Academy of Sciences, Beijing 100049, China \\ ${ }^{4}$ Institute for Advanced Simulation, Institut für Kernphysik and Jülich Center for Hadron Physics, \\ Forschungszentrum Jülich, D-52425 Jülich, Germany \\ ${ }^{5}$ Tbilisi State University, 0186 Tbilisi, Georgia
}

(Received 1 April 2019; published 3 June 2019)

\begin{abstract}
The chiral symmetry of QCD requires energy-dependent pionic strong interactions at low energies. This constraint, however, is not fulfilled by the usual Breit-Wigner parametrization of pionic resonances, leading to masses larger than the real ones. We derive relations between nonleptonic three-body decays of the $B$ meson into a $D$ meson and a pair of light pseudoscalar mesons based on SU(3) chiral symmetry. Employing effective field theory methods, we demonstrate that taking into account the final-state interactions, the experimental data of the decays $B^{-} \rightarrow D^{+} \pi^{-} \pi^{-}, B_{s}^{0} \rightarrow \bar{D}^{0} K^{-} \pi^{+}, B^{0} \rightarrow \bar{D}^{0} \pi^{-} \pi^{+}, B^{-} \rightarrow D^{+} \pi^{-} K^{-}$, and $B^{0} \rightarrow \bar{D}^{0} \pi^{-} K^{+}$can all be described by the nonperturbative $\pi / \eta / K-D / D_{s}$ scattering amplitudes previously obtained from a combination of chiral effective field theory and lattice QCD calculations. The results provide strong support of the scenario that the broad scalar charmed meson $D_{0}^{*}(2400)$ should be replaced by two states, the lower one of which has a mass of around $2.1 \mathrm{GeV}$, much smaller than that extracted from experimental data using a Breit-Wigner parametrization.
\end{abstract}

DOI: 10.1103/PhysRevD.99.114002

\section{INTRODUCTION}

Solving quantum chromodynamics (QCD), the fundamental theory of the strong interaction, is one of the most urgent and challenging tasks in contemporary physics. In principle, the hadronic spectrum is determined by QCD. However, the high nonlinearity of QCD at low energies does not allow for analytical solutions. A direct and clear connection between the fundamental theory and the observed hadronic spectrum is so far absent. Studies of the hadronic spectrum are particularly important in testing the fundamental theory. Therefore, establishing the correct spectroscopy plays an essential role in understanding how the fundamental theory, whose degrees of freedom are quarks and gluons, produces the hadronic spectrum.

\footnotetext{
du@hiskp.uni-bonn.de

fkguo@itp.ac.cn

meissner@hiskp.uni-bonn.de
}

Published by the American Physical Society under the terms of the Creative Commons Attribution 4.0 International license. Further distribution of this work must maintain attribution to the author(s) and the published article's title, journal citation, and DOI. Funded by SCOAP .
The hadronic spectrum has received renewed interest with the recently collected vast amount of experimental data for hadronic processes. In particular, many resonant structures were observed, and they could be the so-called exotic states which cannot be accommodated in the conventional quark model. The lightest scalar $\left(J^{P}=0^{+}\right)$charmstrange meson $D_{s 0}^{*}(2317)$ [1] and the axial-vector $\left(J^{P}=1^{+}\right)$one $D_{s 1}(2460)$ [2] have attracted much attention as they are significantly lighter than the predictions from the quark model calculations, which are around 2.48 and $2.55 \mathrm{GeV}$ [3-5], respectively. It is also noticed that the mass difference between the two states is equal to that between the ground-state pseudoscalar $D^{+}$and the vector $D^{*+}$ within $2 \mathrm{MeV}$. Since attempts to adjust the quark model to adapt the two new states were at odds with previous expectations and raised new puzzles [6], various theoretical investigations were stimulated to unravel their nature. The models proposed for them include $D^{(*)} K$ molecules [7-11], tetraquarks [12,13], a mixture of $c \bar{q}$ with tetraquarks [14], and a cousin of the light scalar mesons [15]. The situation became more obscure by the subsequent observations of the scalar and axial-vector charm-nonstrange mesons, $D_{0}^{*}(2400)$ [16,17] and $D_{1}(2430)$ [16] in 2004. Their quantum numbers indicate that they should be the SU(3) 
partners of the $D_{s 0}^{*}(2317)$ and $D_{s 1}(2460)$, respectively, which sets the starting point for various theoretical studies; see, e.g., Refs. [18-20]. However, this assignment immediately raises a puzzle: Why are the masses of the two strange mesons, $D_{s 0}^{*}(2317)$ and $D_{s 1}(2460)$, almost equal to or even lower than their nonstrange siblings, i.e., the $D_{0}^{*}(2400)$ and $D_{1}(2430)$ ?

While the mass of $D_{s 0}^{*}(2317)$ is well measured and its width is very narrow [21], the situation for the $D_{0}^{*}(2400)$ is obscure. Its width is very broad and its mass has a significant spread in different experimental measurements, e.g., from $2297 \pm 8 \pm 5 \pm 19 \mathrm{MeV}$ at $B A B A R$ [22] to $2403 \pm 14 \pm 35 \mathrm{MeV}$ at FOCUS [17]. In the potential quark model, it would correspond to the lowest $c \bar{q} P$-wave state with a predicted mass of about $2.4 \mathrm{GeV}[3,4]$, which is larger than the averaged mass $2318 \pm 29 \mathrm{MeV}$ in the review of particle physics (RPP) by the particle data group (PDG) [21]. One reason why the analyses that led to the resonance parameters of the $D_{0}^{*}(2400)$ and $D_{1}(2430)$ in Refs. [16,17,22-24], used in the PDG average, should be questioned is that the amplitudes used were inconsistent with the constraints from the chiral symmetry of QCD, which, as a consequence of the Goldstone theorem, requires energy-dependent couplings for the pionic couplings. The standard Breit-Wigner (BW) parametrizations used in the experimental analyses correspond to using constant vertices, and lead to masses larger than their real values. Moreover, the signal ranges for these two states overlap with higher $S$-wave thresholds $\left(D^{(*)} \eta, D_{s}^{(*)} \bar{K}\right)$ that need to be considered in a sound analysis. Fortunately, a theoretical framework satisfying such requirements is provided by the chiral perturbation theory (ChPT) for charmed mesons extended with a unitarization procedure; see, e.g., Refs. [10,11,25-34].

In Ref. [35] it is demonstrated that in the scenario where the lightest scalar (axial-vector) charmed mesons owe their existence to the nonperturbative $\pi / \eta / K-D^{(*)} / D_{s}^{(*)}$ scattering, all of the above-mentioned puzzles in charmed meson spectroscopy get resolved. The $D_{s 0}^{*}(2317)$ and $D_{s 1}(2460)$ are dominantly $D K$ and $D^{*} K$ hadronic molecules (reviewed in Ref. [36]), respectively; heavy-quark spin symmetry predicts that their binding energies are independent of the heavy meson spin. Most importantly, the ordering of the lightest strange, i.e., $D_{s 0}^{*}(2317)$, and the nonstrange scalar, i.e., $D_{0}^{*}(2400)$, becomes natural. The broad $D_{0}^{*}(2400)$ listed in the RPP [21] should be replaced by two states with the lighter one located more than $100 \mathrm{MeV}$ below its strange sibling, i.e., $D_{s 0}^{*}(2317)[35,37]$, as well as the masses of $D_{0}^{*}(2400)$ listed in the RPP [21], which were extracted from BW parametrizations. Furthermore, it was demonstrated that the amplitudes for Goldstone boson $(\phi)-D^{(*)}$ scattering determined by combining unitarized ChPT with lattice QCD in Ref. [30] are fully consistent the recent high-quality $\mathrm{LHCb}$ data of the angular moments for the decay $B^{-} \rightarrow D^{+} \pi^{-} \pi^{-}$[38]. In particular, that the $\mathrm{LHCb}$ data can be fitted with an amplitude having two $D_{0}^{*}$ states, none of whose masses agree with that of the experimental extractions for the $D_{0}^{*}(2400)$, means that the resonance parameters for the scalar charm-nonstrange meson need to be reconsidered by the PDG and by the community.

As the final-state interaction (FSI) between the two $\pi^{-}$ is negligible since they are in an isospin-two state, the reaction $B^{-} \rightarrow D^{+} \pi^{-} \pi^{-}$[38] provides great access to the $D \pi$ system and thus to the charm-nonstrange mesons. The energy range of the $D_{0}^{*}(2400)$ overlaps with two $S$-wave thresholds ( $D \eta$ and $D_{s} \bar{K}$ ), and thus these channels need to be considered and leave their imprints on observables [35]. It means that all channels, i.e., $D^{+} \pi^{-}$, $D^{0} \pi^{0}, D^{0} \eta$, and $D_{s}^{+} K^{-}$, coupled to the $D^{+} \pi^{-}$need to be taken into account in the intermediate states for describing the $D^{+} \pi^{-}$distributions $\lesssim 2.5 \mathrm{GeV}$ of the decay $B^{-} \rightarrow D^{+} \pi^{-} \pi^{-}$. The $D^{0}, D^{+}$, and $D_{s}^{+}$mesons form a $\overline{3}$ representation of the light-quark $\mathrm{SU}(3)$ flavor symmetry. Using the $\mathrm{SU}(3)$ transformation properties of the effective Hamiltonian for weak nonleptonic $B$-meson three-body decays, a ratio between the four channels can be derived $[35,39]$. Because the two $\pi^{-}$could only be in a state where the relative angular momentum is even, the effective Lagrangian for the weak decays of $\bar{B}$ to $D$ induced by the Cabibbo-allowed transition $b \rightarrow c \bar{u} d$, with the emission of two symmetrized light pseudoscalar mesons was constructed in the Appendix of Ref. [35]. In this paper, we extend the effective weak Lagrangian to include the Cabibbo-suppressed decays with two nonidentical light pseudoscalar mesons. Since we will focus on the energy region only a few hundred $\mathrm{MeV}$ above the corresponding thresholds, one of the light pseudoscalar mesons would move fast. For simplicity, we do not consider the FSIs from crossed channels, i.e., the interaction between $D / D_{s}$ and the fast-moving light pseudoscalar meson and the interaction between the two light pseudoscalar mesons. As in the isobar model, the crossed-channel effects are simply assumed to be encoded in an extra complex factor which would be fixed by fitting to experimental data. However, the FSI between the charmed meson and the soft light pseudoscalar meson and that from coupled channels are taken into account.

While the weak production vertices can be derived from the weak effective Lagrangian, the rescatterings between the charmed mesons and the soft light pseudoscalar mesons are described by the unitarized ChPT; see, e.g., in Refs. [29-32,34]. In Ref. [30], the low-energy constants (LECs) of ChPT for charmed mesons, and thus the chiral amplitudes for the $D-\phi$ scattering, are obtained by fitting to a lattice QCD calculation of the scattering lengths for the channels with connected Wick contractions only, i.e., $D \pi$ with isospin $I=3 / 2, D \bar{K}$ with $I=0$ and $1, D_{s} K$ and $D_{s} \pi$. With the parameters fixed in Ref. [30], the predicted energy levels for the coupled-channel systems in a finite volume 
are found $[37,40]$ to be in remarkable agreement with recent lattice QCD calculations $[41,42] .{ }^{1}$ It indicates the reliability of the well-constrained chiral amplitudes in Ref. [30]. As in Ref. [35], we will demonstrate that the amplitudes obtained in Ref. [30] are consistent with the experimental data on the three-body decays $B_{s}^{0} \rightarrow \bar{D}^{0} K^{-} \pi^{+}$, $B^{0} \rightarrow \bar{D}^{0} \pi^{-} \pi^{+}, B^{-} \rightarrow D^{+} \pi^{-} K^{-}$, and $B^{0} \rightarrow \bar{D}^{0} \pi^{-} K^{+}$, and try to determine the LECs of the weak effective Lagrangian from these decays.

This paper is organized as follows. In Sec. II, we will briefly discuss the implication of the chiral symmetry on pionic BW resonances. In Sec. III, the chiral effective weak Lagrangian describing the $B$-meson decays to the $D$ meson associated with two light pseudoscalar mesons is constructed. The decay amplitudes satisfying unitarity for the reactions $B_{s}^{0} \rightarrow \bar{D}^{0} K^{-} \pi^{+}, B^{0} \rightarrow \bar{D}^{0} \pi^{-} \pi^{+}, B^{-} \rightarrow D^{+} \pi^{-} K^{-}$, and $B^{0} \rightarrow \bar{D}^{0} \pi^{-} K^{+}$are derived in Sec. IV. In Sec. V, the socalled angular moments are calculated and fitted to experimental data. Finally, Sec. VI comprises a brief summary.

\section{IMPLICATION OF CHIRAL SYMMETRY ON PIONIC RESONANCES}

The small masses of the $u, d$, and $s$ quark compared with the QCD scale $\Lambda_{\mathrm{QCD}}$ induce an approximate $\mathrm{SU}(3)_{L} \times$ $\mathrm{SU}(3)_{R}$ chiral symmetry for the strong interactions. As the chiral symmetry is spontaneously broken to its diagonal subgroup $\mathrm{SU}(3)_{V}$, the $\pi, K$, and $\eta$ mesons arise as the pseudo-Goldstone bosons. At the leading order (LO) in the chiral expansion, the interactions of Goldstone bosons with themselves and with matter fields are of a derivative form and thus energy dependent. However, the standard BW parametrization used in the experimental analyses to extract the resonance parameters (mass and width) corresponds to a constant interaction for $S$-wave vertices. Were the energydependent interaction induced by the derivative coupling considered, the mass fitted using a normal BW parametrization would be shifted to a lower value. This point is illustrated in the following.

For simplicity, we neglect the energy dependence of the decay width in this section. The BW parametrization for a resonance in the $D \pi S$-wave reads

$$
F_{0}(s) \propto \frac{1}{s-m_{0}^{2}+i m_{0} \Gamma},
$$

where the $m_{0}$ and $\Gamma$ represent the BW mass and width of the resonance, respectively. Now let us calculate the peak position of this $\mathrm{BW}$ parametrization. It corresponds to the energy where

\footnotetext{
${ }^{1}$ In an updated fit [34] to the lattice data, several different fits were found, and the parameters in the set which can reproduce the $D_{s 0}^{*}(2317)$ mass as well as satisfy the $N_{c}$ scaling are similar to those determined in Ref. [30].
}

$$
\frac{d}{d s}\left|F_{0}(s)\right|^{2} \propto-\frac{2\left(s-m_{0}^{2}\right)}{\left[\left(s-m_{0}^{2}\right)^{2}+m_{0}^{2} \Gamma^{2}\right]^{2}}=0,
$$

which means that the BW mass for a resonance corresponds to the value of the peak position. In order to take the chiral symmetric constraint into account, one may simply modify Eq. (1) by introducing a pion energy factor as

$$
F_{0}^{\prime}(s) \propto \frac{E_{\pi}}{s-m_{0}^{2}+i m_{0} \Gamma}
$$

with $E_{\pi}=\left(s+M_{\pi}^{2}-M_{D}^{2}\right) /(2 \sqrt{s})$ the energy of the produced soft pion in the rest frame of the $D \pi$ system. The peak position $s_{\text {peak }}$ can be obtained with the same approach, that is

$$
\left.\frac{d}{d s}\left|F_{0}^{\prime}(s)\right|^{2}\right|_{s=s_{\text {peak }}}=0
$$

It is easy to see that the peak position $s_{\text {peak }}$ is shifted from $m_{0}^{2}$. The shift is expected to be small compared with $m_{0}$ for $\Gamma \ll m_{0}$. Let $s_{\text {peak }}=\left(m_{0}+\Delta\right)^{2}$. Keeping only the term linear in $\Delta$, one gets

$\Delta \simeq \frac{\Gamma^{2}\left(m_{0}^{2}-M_{\pi}^{2}+M_{D}^{2}\right)}{2 m_{0}\left[2\left(m_{0}^{2}+M_{\pi}^{2}-M_{D}^{2}\right)-\Gamma^{2}\right]}=\frac{\Gamma^{2} E_{D}}{4 m_{0} E_{\pi}-\Gamma^{2}}$,

where $E_{D}$ is the energy of the produced $D$ in the rest frame of the $D \pi$ system with a total energy $m_{0}$. Thus, for the case $4 m_{0} E_{\pi}>\Gamma^{2}$, e.g., that of the $D_{0}^{*}(2400)$, the shift $\Delta$ is positive, which means that the mass of the resonance is lower than the peak position.

Nevertheless, the modification in Eq. (2) can only be applied in a small energy region before the coupled-channel effect becomes important, and thus is neither practical nor systematic. A theoretical framework satisfying both the chiral symmetry constraint and unitarity taking into account coupled channels is needed. Such requirements are fulfilled by the unitarized ChPT; see, e.g., Refs. [43-47].

\section{CHIRAL EFFECTIVE LAGRANGIAN}

While the ground-state pseudoscalar octet $(\phi)$ at low energies can be treated as the pseudo-Goldstone bosons associated with the spontaneous breaking of the approximate chiral symmetry into its diagonal subgroup $\mathrm{SU}(3)_{V}, \mathrm{SU}(3)_{V}$ is realized in the Wigner-Weyl mode. It means that the nonGoldstone bosons (matter fields) form multiplets which correspond to different irreducible representations of $\mathrm{SU}(3)$. In particular, the $B^{-}, \bar{B}^{0}$, and $\bar{B}_{s}^{0}$ mesons, and $D^{0}$, $D^{+}$, and $D_{s}^{+}$mesons form two $\overline{3}$ fundamental representations of SU(3). In the language of the Callan-Coleman-WessZumino formalism [48,49], the Goldstone bosons are realized nonlinearly in $u(x)$, which transforms under a global chiral transformation $g_{L} \times g_{R} \in \mathrm{SU}(3)_{L} \times \mathrm{SU}(3)_{R}$ as 


$$
u(x) \mapsto g_{R} u(x) h(x)^{\dagger}=h(x) u(x) g_{L}^{\dagger},
$$

where the compensator field $h(x)$ is a nonlinear function of $g_{L}, g_{R}$, and $u(x)$, and it reduces to $h(x)=g_{L}=g_{R}$, independent of $u(x)$, for an $\mathrm{SU}(3)_{V}$ transformation. We parametrize $u$ as $u=\exp \left(i \phi /\left(\sqrt{2} F_{0}\right)\right)$, where $F_{0}$ is the pion decay constant in the chiral limit and

$$
\phi=\left(\begin{array}{ccc}
\frac{1}{\sqrt{2}} \pi^{0}+\frac{1}{\sqrt{6}} \eta & \pi^{+} & K^{+} \\
\pi^{-} & -\frac{1}{\sqrt{2}} \pi^{0}+\frac{1}{\sqrt{6}} \eta & K^{0} \\
K^{-} & \bar{K}^{0} & -\frac{2}{\sqrt{6}} \eta
\end{array}\right) .
$$

From the chiral transformation properties of $u(x)$, one can derive the axial-vector $u_{\mu}$ and vector $\Gamma_{\mu}$ currents $[48,49]^{2}$ :

$$
\begin{aligned}
u_{\mu} & =i\left(u^{\dagger} \partial_{\mu} u-u \partial_{\mu} u^{\dagger}\right), \\
\Gamma_{\mu} & =\frac{1}{2}\left(u^{\dagger} \partial_{\mu} u+u \partial_{\mu} u^{\dagger}\right) .
\end{aligned}
$$

The axial-vector $u_{\mu}$ transforms homogeneously,

$$
u_{\mu} \mapsto h u_{\mu} h^{\dagger}
$$

whereas $\Gamma_{\mu}$ transforms inhomogeneously,

$$
\Gamma_{\mu} \mapsto h \Gamma_{\mu} h^{\dagger}+h \partial_{\mu} h^{\dagger}
$$

The transformation properties of the matter fields are not unique. It is, however, convenient to construct the triplets for the $B$ and $D$ mesons such that they transform under $g_{L} \times g_{R}$ as

$$
B \mapsto B h^{\dagger}, \quad D \mapsto D h^{\dagger},
$$

where we have introduced the notation

$$
B=\left(B^{-}, \bar{B}^{0}, \bar{B}_{s}^{0}\right) \quad \text { and } \quad D=\left(D^{0}, D^{+}, D_{s}^{+}\right) .
$$

In particular, the quantity $\Gamma_{\mu}$ transforms precisely as a gauge $\mathrm{SU}(3)_{V}$ transformation. With it we can construct a covariant derivative for a matter field, e.g.,

$$
\nabla_{\mu} D^{\dagger}=\partial_{\mu} D^{\dagger}+\Gamma_{\mu} D^{\dagger} .
$$

To construct the effective Lagrangian, one has to specify the power-counting rules. At low energies, the momenta as well as the masses of Goldstone bosons are counted as $\mathcal{O}(p)$ [52]. However, the nonvanishing mass of the matter field in the chiral limit introduces new energy scales (here $M_{D}$ and $M_{B}$ ) which are larger than the hard chiral scale

\footnotetext{
${ }^{2}$ The inclusion of the external currents is straightforward; see Refs. [50,51].
}

$\Lambda_{\chi} \sim 4 \pi F_{0}$. Therefore, the temporal component of the momenta of $D$ and $B$ mesons should be counted as $\mathcal{O}(1)$; see, e.g., Refs. [28,51,53]. Yet, the small threemomenta of the $D$ and $B$ mesons are counted as $\mathcal{O}(p)$. To incorporate the explicit chiral breaking due to the nonvanishing light-quark masses, a spurion $\chi_{ \pm}$is introduced as

$$
\chi_{ \pm}=u^{\dagger} \chi u^{\dagger} \pm u \chi^{\dagger} u,
$$

with

$$
\chi=2 B_{0} \operatorname{diag}\left(m_{u}, m_{d}, m_{s}\right),
$$

where $B_{0}=|\langle 0|\bar{q} q| 0\rangle| / F_{0}^{2}$ is a constant related to the quark condensate. The spurion transforms under chiral symmetry as

$$
\chi_{ \pm} \mapsto h \chi_{ \pm} h^{\dagger}
$$

The power-counting rules for the building blocks of the effective Lagrangian are

$$
\begin{aligned}
\nabla_{\mu} D^{\dagger} & \sim \mathcal{O}(1), & & \nabla_{\mu} B^{\dagger} \sim \mathcal{O}(1), \\
u_{\mu} & \sim \mathcal{O}(p), & & \chi_{ \pm} \sim \mathcal{O}\left(p^{2}\right) .
\end{aligned}
$$

With the above transformation properties and the powercounting rules, one can construct the effective chiral Lagrangian for $D-\phi$ interactions; e.g., Refs. [26,28-31,54].

At low energies, for the processes with $\Delta b=1$ and $\Delta c=1$ the interaction can be described by the effective weak Hamiltonian $H_{\text {eff }}$ which at $\mathrm{LO}$ has the form

$H_{\text {eff }}=\frac{G_{F}}{\sqrt{2}} V_{c b}^{*} V_{u d}\left(C_{1} \mathcal{O}_{1}^{d}+C_{2} \mathcal{O}_{2}^{d}\right)+(b \rightarrow s)+$ H.c.,

with $G_{F}$ the Fermi constant, $V_{i j}$ the Cabibbo-KobayashMaskawa matrix elements, and $C_{i}$ the scale-dependent Wilson coefficients. Here, the tree-level operators read

$$
\begin{aligned}
& \mathcal{O}_{1}^{d}=\left(\bar{c}_{a} b_{b}\right)_{L}\left(\bar{d}_{b} u_{a}\right)_{L}, \\
& \mathcal{O}_{2}^{d}=\left(\bar{c}_{a} b_{a}\right)_{L}\left(\bar{d}_{b} u_{b}\right)_{L},
\end{aligned}
$$

with the subscripts $a$ and $b$ the color indices. The subscript $L$ indicates that only the left-hand components of the quarks are involved. Note that here the color space is irrelevant for our discussion; thus we simply drop the subscripts of $C_{i}$ and $\mathcal{O}_{i}$ hereafter. One can make the effective Hamiltonian fully chirally invariant by introducing a spurion $H_{i}^{j}$ transforming as [55]

$$
H_{i}^{j} \mapsto H_{i^{\prime}}^{j^{\prime}}\left(g_{L}\right)_{i}^{i^{\prime}}\left(g_{L}^{\dagger}\right)_{j^{\prime}}^{j}
$$


Then the new Hamiltonian

$$
H_{\mathrm{eff}}^{\prime}=\frac{G_{F}}{\sqrt{2}} V_{c b}^{*} V_{u d} H_{i}^{j} C(\bar{c} b)_{L}\left(\bar{q}_{L}^{i} q_{L j}\right)
$$

is chirally invariant. For Eq. (16), the spurion $H_{i}^{j}$ (the lower index labels rows and the upper one labels columns) corresponds to

$$
H=\left(\begin{array}{ccc}
0 & 0 & 0 \\
1 & 0 & 0 \\
V_{u s} / V_{u d} & 0 & 0
\end{array}\right) .
$$

Here, $V_{u s} / V_{u d}$ is nothing but $-\sin \theta_{1}$ (to be written as $-s_{1}$ for simplicity) with $\theta_{1}$ the Cabbibo angle. Then the component $H_{2}^{1}$ describes the Cabibbo-allowed decays and $H_{3}^{1}$ the Cabibbo-suppressed ones. In the matrix form, $H$ transforms under chiral symmetry as

$$
H \mapsto g_{L} H g_{L}^{\dagger} .
$$

It is more convenient to introduce a homogeneously transforming spurion as

$$
t=u H u^{\dagger}
$$

With those ingredients, we are prepared to construct the effective Lagrangian describing the three-body nonleptonic decays of $B$ mesons to $D$ mesons and two light pseudoscalars. We are interested in the region of the invariant mass of a pair of the $D$ and one pseudoscalar not far from their threshold, such that this light pseudoscalar can be safely treated as a soft Goldstone boson, while the other one moves fast and can be treated as a matter field rather than a Goldstone boson. The fast-moving pseudoscalar is realized linearly in a matrix form $M$ transforming as

$$
M \mapsto h M h^{\dagger},
$$

and it has the same form as $\phi$, i.e.,

$$
M=\left(\begin{array}{ccc}
\frac{1}{\sqrt{2}} \pi^{0}+\frac{1}{\sqrt{6}} \eta & \pi^{+} & K^{+} \\
\pi^{-} & -\frac{1}{\sqrt{2}} \pi^{0}+\frac{1}{\sqrt{6}} \eta & K^{0} \\
K^{-} & \bar{K}^{0} & -\frac{2}{\sqrt{6}} \eta
\end{array}\right) .
$$

Consequently, utilizing the power counting in Eq. (14), chiral symmetry implies that the effective Lagrangian at $\mathcal{O}(p)$ has the form of $[35,39]$

$$
\begin{aligned}
\mathcal{L}_{\text {eff }}= & B\left[c_{1}\left(u_{\mu} t M+M t u_{\mu}\right)+c_{2}\left(u_{\mu} M+M u_{\mu}\right) t+c_{3} t\left(u_{\mu} M+M u_{\mu}\right)+c_{4}\left(u_{\mu}\langle M t\rangle+M\left\langle u_{\mu} t\right\rangle\right)+c_{5} t\left\langle M u_{\mu}\right\rangle\right. \\
& \left.+c_{6}\left\langle\left(M u_{\mu}+u_{\mu} M\right) t\right\rangle\right] \nabla^{\mu} D^{\dagger}+B\left[d_{1}\left(u_{\mu} t M-M t u_{\mu}\right)+d_{2}\left(u_{\mu} M-M u_{\mu}\right) t+d_{3} t\left(u_{\mu} M-M u_{\mu}\right)\right. \\
& \left.+d_{4}\left(u_{\mu}\langle M t\rangle-M\left\langle u_{\mu} t\right\rangle\right)+d_{6}\left\langle\left(M u_{\mu}-u_{\mu} M\right) t\right\rangle\right] \nabla^{\mu} D^{\dagger},
\end{aligned}
$$

where the $c_{i}$ and $d_{i}$ are LECs, and $\langle\ldots\rangle$ denotes the traces in the SU(3) light-flavor space. Note that the momentum operator $\nabla_{\mu}$ in Eq. (25) is chosen to act on the charmed meson field $D$. It could be on $B$ (or $M$ ) independently as well. However, in our case, $M_{B} \gg M_{D}+2 M_{\pi}$ and $M_{D} \gg$ $M_{\pi}$ imply that they would produce the same structures up to the LO and we can combine them by redefining the LECs in the heavy meson limit. This effective Lagrangian considers both the chiral symmetry and flavor SU(3) constraints (the latter has been considered in Ref. [39]). Finally, we divide the Lagrangian (25) into two groups which are symmetric and antisymmetric in the two light pseudoscalars corresponding to the cases where the relative orbital angular momentum of the light pseudoscalars pair is even and odd, respectively [39].

\section{B-MESON THREE-BODY DECAY AMPLITUDES}

The nonleptonic $B$-meson three-body decays $B \rightarrow D \phi M$, where $M$ denotes the fast-moving light pesudoscalar and $\phi$ denotes the soft one as in the last section, provide access to the $D-\phi$ interaction via FSI. ${ }^{3}$ From now on, we write the decay product in the ordering that the charmed meson is followed by the soft and hard light pseudoscalar subsequently. The FSI between the $D$ and the hard $M$ and that between $M$ and $\phi$, which are not expected to produce any nontrivial structure sensitive to the energy variation, are encoded into an extra complex factor for simplicity. The Feynman diagrams for the decay $B \rightarrow D \phi M$ are shown in Fig. 1, where the square denotes the FSI between $D$ and $\phi$.

The decay amplitude projected into the $D \phi$ channel at low energies can be decomposed into $S, P$, and $D$ waves (corresponding to the orbital angular momentum of the $D \phi$ pair being $L=0, L=1$, and $L=2$, respectively), ${ }^{4}$

\footnotetext{
${ }^{3}$ We are only interested in the energy region where the $D$ and one of the light pseudoscalars have an invariant mass not far from their threshold.

${ }^{4}$ Here, we neglect the partial waves with $L \geq 3$, which is perfectly fine in the energy region of interest as shown in the experimental data; see, e.g., Refs. [23,24,38,56,57].
} 


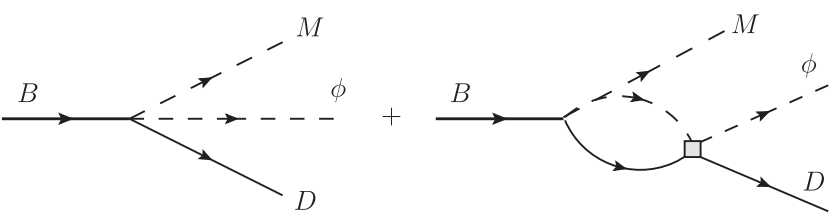

FIG. 1. Illustrative graphs for the decay $B \rightarrow D \phi M$. The square denotes the FSI between the $D$ meson and the Goldstone boson. In the loop, all coupled channels connecting the initial to the final states contribute.

$$
\begin{aligned}
\mathcal{A}(B \rightarrow D \phi M)= & \mathcal{A}_{0}(s)+\sqrt{3} \mathcal{A}_{1}(s) P_{1}(z) \\
& +\sqrt{5} \mathcal{A}_{2}(s) P_{2}(z),
\end{aligned}
$$

where $\mathcal{A}_{0,1,2}(s)$ correspond to the partial-wave decomposed amplitudes for $D \phi$ in the $S, P$, and $D$ waves, respectively, and $P_{L}(z)$ are the Legendre polynomials with $z$ the cosine of the helicity angle of the $D \phi$ system, i.e., the angle between the moving directions of the $\phi$ and the $M$ in the $D \phi$ rest frame. For the $P$ and $D$ waves, the resonances are relatively narrow and thus it is reasonable to parametrize them by BW amplitudes. For the $S$ wave, we calculate the diagrams in Fig. 1 with the effective Lagrangian (25) with the FSI provided by the $D \phi$ interaction determined in Ref. [30] with the coupled-channel effects taken into account.

For the decay $B^{-} \rightarrow D^{+} \pi^{-} \pi^{-}$, the relative orbital angular momenta of the two light mesons could only be even, which correspond to the first term of the Lagrangian in Eq. (25), i.e., the terms with the LECs $c_{i}$. With the Lagrangian in Eq. (25), the production vertices for the possible intermediate states $D^{0} \pi^{0}, D^{+} \pi^{-}, D^{0} \eta$, and $D_{s}^{+} K^{-}$are listed in Table I.

In the heavy-quark limit, $p_{D} \cdot p_{\phi}=M_{D} E_{\phi}$, with $E_{\phi}$ the energy of $\phi$ in the rest frame of $D \phi$. For convenience, we introduce two parameters $A$ and $B$ [35]

$A=\frac{\sqrt{2}}{F_{0}}\left(c_{1}+c_{4}\right) M_{D}, \quad B=\frac{2 \sqrt{2}}{3 F_{0}}\left(c_{2}+c_{6}\right) M_{D}$.

Taking the FSI into account, the $S$-wave decay amplitude for $B^{-} \rightarrow D^{+} \pi^{-} \pi^{-}$reads

$$
\begin{aligned}
\mathcal{A}_{0}(s)= & 2 A E_{\pi}+2 A E_{\pi} G_{D \pi}(s) T_{D^{+} \pi^{-} \rightarrow D^{+} \pi^{-}}(s) \\
& +\frac{A}{\sqrt{2}} E_{\pi} G_{D \pi}(s) T_{D^{0} \pi^{0} \rightarrow D^{+} \pi^{-}}(s) \\
& +\frac{A+3 B}{\sqrt{6}} E_{\eta} G_{D \eta}(s) T_{D^{0} \eta \rightarrow D^{+} \pi^{-}}(s) \\
& +A E_{K} G_{D_{s} \bar{K}}(s) T_{D_{s}^{+} K^{-} \rightarrow D^{+} \pi^{-}}(s),
\end{aligned}
$$

where $s$ is the center-of-mass energy squared of the $D \phi$ system, and $G_{D \phi}(s)$ is the loop function (the "fundamental bubble") depicted in Fig. 1 coupling to the channel $D \phi$. Unitarity relates the imaginary part of the loop function $G_{D \phi}(s)$ with the $D \phi$ two-body phase factor
TABLE I. Production vertices for the possible intermediate states contributing to $B^{-} \rightarrow D^{+} \pi^{-} \pi^{-}$. Here, $p_{D_{(s)}}$ and $p_{\phi}$ denote the four-momenta of the charmed meson and the Goldstone boson, respectively.

\begin{tabular}{lc}
\hline \hline Process & Production amplitude \\
\hline$B^{-} \rightarrow D^{0} \pi^{0} \pi^{-}$ & $\frac{1}{F_{0}}\left(c_{1}+c_{4}\right) p_{D} \cdot p_{\pi}$ \\
$B^{-} \rightarrow D^{0} \eta \pi^{-}$ & $\frac{1}{\sqrt{3} F_{0}}\left(c_{1}+c_{4}+2 c_{2}+2 c_{6}\right) p_{D} \cdot p_{\eta}$ \\
$B^{-} \rightarrow D^{+} \pi^{-} \pi^{-}$ & $\frac{2 \sqrt{2}}{F_{0}}\left(c_{1}+c_{4}\right) p_{D} \cdot p_{\pi}$ \\
$B^{-} \rightarrow D_{s}^{+} K^{-} \pi^{-}$ & $\frac{\sqrt{2}}{F_{0}}\left(c_{1}+c_{4}\right) p_{D_{s}} \cdot p_{K}$ \\
\hline \hline
\end{tabular}

$\rho(s)=q_{\phi} /(8 \pi \sqrt{s}) \theta\left(s-\left(M_{D}+M_{\phi}\right)^{2}\right)$, with $q_{\phi}$ the magnitude of the $\phi$ three-momentum in the $D \phi$ center-of-mass frame, by $\operatorname{Im} G_{D \phi}(s)=-\rho(s)$, which allows us to represent $G_{D \phi}(s)$ via a once-subtracted dispersion relation

$$
\begin{aligned}
G_{D \phi}(s) & \\
= & \frac{1}{16 \pi^{2}}\left\{a_{A}+\log \frac{M_{D}^{2}}{\mu^{2}}+\frac{M_{\phi}^{2}-M_{D}^{2}+s}{2 s} \log \frac{M_{\phi}^{2}}{M_{D}^{2}}\right. \\
& +\frac{\sigma}{2 s}\left[\log \left(s-M_{D}^{2}+M_{\phi}^{2}+\sigma\right)-\log \left(-s+M_{D}^{2}-M_{\phi}^{2}+\sigma\right)\right. \\
& \left.\left.+\log \left(s+M_{D}^{2}-M_{\phi}^{2}+\sigma\right)-\log \left(-s-M_{D}^{2}+M_{\phi}^{2}+\sigma\right)\right]\right\},
\end{aligned}
$$

with $a_{A}$ a scale-dependent subtraction constant, $\sigma=\{[s-$ $\left.\left.\left(M_{D}+M_{\phi}\right)^{2}\right]\left[s-\left(M_{D}-M_{\phi}\right)^{2}\right]\right\}^{1 / 2}$ and $\mu$ the scale of dimensional regularization. The subtraction $a_{A}$ is related to the renormalization of the interaction vertices and varies for different processes. A change of $\mu$ can be absorbed into a corresponding change of $a_{A}$, and we will take $\mu=1 \mathrm{GeV}$ as in Ref. [30].

The amplitudes for the FSI can be expressed in the isospin basis. While $D^{+} \pi^{-}$can be decomposed into isospin $I=1 / 2$ and $3 / 2$ systems, $D^{+} \eta$ and $D_{s}^{+} K^{-}$can only form $I=1 / 2$. The relations between the isospin basis and physical particle basis are given by $[29,31]^{5}$

$$
\begin{aligned}
T_{D^{0} \pi^{0} \rightarrow D^{+} \pi^{-}} & =-\frac{\sqrt{2}}{3} T_{D \pi \rightarrow D \pi}^{3 / 2}+\frac{\sqrt{2}}{3} T_{D \pi \rightarrow D \pi}^{1 / 2}, \\
T_{D^{0} \eta \rightarrow D^{+} \pi^{-}} & =\sqrt{\frac{2}{3}} T_{D \eta \rightarrow D \pi}^{1 / 2}, \\
T_{D^{+} \pi^{-} \rightarrow D^{+} \pi^{-}} & =\frac{1}{3} T_{D \pi \rightarrow D \pi}^{3 / 2}+\frac{2}{3} T_{D \pi \rightarrow D \pi}^{1 / 2}, \\
T_{D_{s}^{+} K^{-} \rightarrow D^{+} \pi^{-}} & =\sqrt{\frac{2}{3}} T_{D_{s} \bar{K} \rightarrow D \pi}^{1 / 2},
\end{aligned}
$$

${ }^{5}$ We use the phase convention such that $\left|\pi^{+}\right\rangle=-|1,+1\rangle$, $\left.\bar{K}^{0}\right\rangle=-\left|\frac{1}{2},+\frac{1}{2}\right\rangle$, and $\left|D^{+}\right\rangle=-\left|\frac{1}{2},+\frac{1}{2}\right\rangle$, where the two numbers in $|\ldots\rangle$ on the right-hand side are isospin $I$ and the third component $I_{3}$. 
where the superscripts indicate the total isospin $I$. The amplitudes in the isospin basis can be found in Refs. [29-31]. As a result, we get the $S$-wave decay amplitude for the process $B^{-} \rightarrow D^{+} \pi^{-} \pi^{-}$[35]

$$
\begin{aligned}
\mathcal{A}_{0}(s)= & A E_{\pi}\left[2+G_{D \pi}(s)\left(\frac{5}{3} T_{11}^{1 / 2}(s)+\frac{1}{3} T^{3 / 2}(s)\right)\right] \\
& +\frac{1}{3}(A+3 B) E_{\eta} G_{D \eta}(s) T_{21}^{1 / 2}(s) \\
& +\sqrt{\frac{2}{3}} A E_{K} G_{D_{s} \bar{K}}(s) T_{31}^{1 / 2}(s) .
\end{aligned}
$$

Here, we write the scattering amplitudes in the matrix form $T_{i j}^{I}(s)$ with the total isospin $I$, where $i, j$ are channel indices with 1,2 , and 3 referring to $D \pi, D \eta$, and $D_{s} \bar{K}$, respectively. Note that only two LECs ( $A$ and $B$ ) appear in Eq. (31).

The above amplitude can also be obtained in a different way $[58,59]$ without constructing the effective Lagrangian. At the quark level, the decay $B^{-} \rightarrow D^{+} \pi^{-} \pi^{-}$is mediated by the weak process $b \bar{u} \rightarrow\left(W^{-} c\right) \bar{u} \rightarrow(d \bar{u} c) \bar{u}$. In order to obtain a three-meson final state, an extra $q \bar{q}$ pair must be created from the QCD vacuum. The quantum numbers of vacuum imply that the extra $q \bar{q}$ pair must be an $\mathrm{SU}(3)$ flavor singlet, $u \bar{u}+d \bar{d}+s \bar{s}$. Next we hadronize the $c \bar{u} d \bar{u}(u \bar{u}+$ $d \bar{d}+s \bar{s})$ combination in terms of three pseudoscalar mesons. To this end, we introduce a $q \bar{q}$ matrix $\Phi$ [59],

$$
\begin{aligned}
\Phi & =q \bar{q}=\left(\begin{array}{l}
u \\
d \\
s \\
c
\end{array}\right)\left(\begin{array}{llll}
\bar{u} & \bar{d} & \bar{s} & \bar{c}
\end{array}\right) \\
& =\left(\begin{array}{llll}
u \bar{u} & u \bar{d} & u \bar{s} & u \bar{c} \\
d \bar{u} & d \bar{d} & d \bar{s} & d \bar{c} \\
s \bar{u} & s \bar{d} & s \bar{s} & s \bar{c} \\
c \bar{u} & c \bar{d} & c \bar{s} & c \bar{c}
\end{array}\right)
\end{aligned}
$$

which fulfills

$\Phi \Phi=(q \bar{q})(q \bar{q})=q(\bar{q} q) \bar{q}=(\bar{u} u+\bar{d} d+\bar{s} s+\bar{c} c) \Phi$,

where the singlet $(\bar{q} q)=(\bar{u} u+\bar{d} d+\bar{s} s+\bar{c} c)$ corresponds to a $\bar{q} q$ pair creation from the QCD vacuum. In terms of pseudoscalar mesons, the matrix $\Phi$ has the form of

$\Phi=\left(\begin{array}{cccc}\frac{1}{\sqrt{2}} \pi^{0}+\frac{1}{\sqrt{6}} \eta & \pi^{+} & K^{+} & \bar{D}^{0} \\ \pi^{-} & -\frac{1}{\sqrt{2}} \pi^{0}+\frac{1}{\sqrt{6}} \eta & K^{0} & D^{-} \\ K^{-} & \bar{K}^{0} & -\frac{2}{\sqrt{6}} \eta & D_{s}^{-} \\ D^{0} & D^{+} & D_{s}^{+} & \eta_{c}\end{array}\right)$.
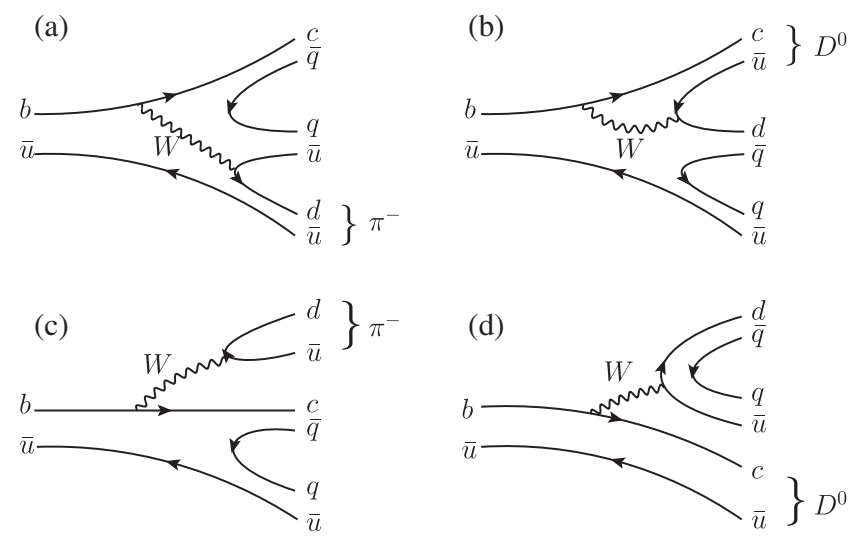

FIG. 2. Schematic representation at the quark level for the decay of the $B^{-}$meson into $D^{0} \pi^{0} \pi^{-} / D^{+} \pi^{-} \pi^{-} / D^{0} \eta \pi^{-} / D_{s}^{+} K^{-} \pi^{-}$. The diagrams (a) and (c) represent hadronization through $[d \overline{(u)}](\Phi \Phi)_{41}$, and the diagrams (b) and (d) represent hadronization through $[c \bar{u})](\Phi \Phi)_{21}$.

The hadronization of $c \bar{u} d \bar{u}(q \bar{q})$ could proceed in two distinct ways, i.e., $[d \bar{u}](\Phi \Phi)_{41}$ and $[c \bar{u}](\Phi \Phi)_{21}$, which correspond to the diagrams $(a, c)$ and $(b, d)$, respectively, in Fig. 2. These two sets of hadronization give two independent structures as

$$
\begin{aligned}
& {[d \bar{u}](\Phi \Phi)_{41}} \\
& \quad=\pi^{-}\left(\frac{1}{\sqrt{2}} D^{0} \pi^{0}+\frac{1}{\sqrt{6}} D^{0} \eta+D^{+} \pi^{-}+D_{s}^{+} K^{-}\right)+\cdots
\end{aligned}
$$

and

$$
[c \bar{u}](\Phi \Phi)_{21}=D^{0}\left(\sqrt{\frac{2}{3}} \eta \pi^{-}\right)+\cdots,
$$

respectively. We assume that the production amplitudes for the diagrams in Fig. 2 are $a, b, c$, and $d$, respectively. Then the amplitudes for the processes in Table I are proportional to the following factors:

$\frac{a+c}{\sqrt{2}}, \frac{(a+c)+2(b+d)}{\sqrt{6}}, \quad 2(a+c)$, and $(a+c)$,

respectively. Compared with the expressions in Table I, we find $A=a+c$ and $B=2(b+d) / 3$. Then the SU(3) flavor structure for the amplitudes of the four processes in Table I reads

$$
\frac{1}{\sqrt{2}} A:\left(\frac{1}{\sqrt{6}} A+\sqrt{\frac{3}{2}} B\right): 2 A: A
$$

Expressed in the isospin basis, the amplitudes for the production of the $D \pi(I=1 / 2), D \pi(I=3 / 2), D \eta(I=$ $1 / 2)$, and $D_{s} \bar{K}(I=1 / 2)$ channels have the ratios 
TABLE II. Weak amplitudes contributing to the decays $B_{s}^{0} \rightarrow \bar{D}^{0} K^{-} \pi^{+}, B^{0} \rightarrow \bar{D}^{0} \pi^{-} \pi^{+}, B^{-} \rightarrow D^{+} \pi^{-} K^{-}$, and $B^{0} \rightarrow \bar{D}^{0} \pi^{-} K^{+}$through coupled-channel effects.

\begin{tabular}{lc}
\hline \hline Processes & Weak production vertices \\
\hline$B_{s}^{0} \rightarrow \bar{D}^{0} K^{-} \pi^{+}$ & $\frac{\sqrt{2} M_{D}}{F_{0}} E_{K}\left(\left(c_{2}+c_{4}\right)+\left(d_{2}+d_{4}\right)\right)$ \\
$B_{s}^{0} \rightarrow D^{-} \bar{K}^{0} \pi^{+}$ & $\frac{\sqrt{2} M_{D}}{F_{0}} E_{K}\left(\left(c_{1}+c_{4}\right)+\left(d_{1}+d_{4}\right)\right)$ \\
$B_{s}^{0} \rightarrow \bar{D}_{s} \eta \pi^{+}$ & $\frac{2 M_{D_{s}}}{\sqrt{3} F_{0}} E_{\eta}\left(\left(c_{6}-c_{4}\right)-d_{4}\right)$ \\
$B_{s}^{0} \rightarrow \bar{D}_{s} \pi^{0} \pi^{+}$ & $\frac{2 M_{D_{s}}}{F_{0}} E_{\pi} d_{6}$ \\
$B^{0} \rightarrow \bar{D}^{0} \pi^{-} \pi^{+}$ & $\frac{\sqrt{2} M_{D}}{F_{0}} E_{\pi}\left(\left(c_{2}+c_{3}+c_{4}+2 c_{5}\right)+\left(d_{2}-d_{3}+d_{4}\right)\right)$ \\
$B^{0} \rightarrow D^{-} \eta \pi^{+}$ & $\frac{M_{D}}{\sqrt{3} F_{0}} E_{\eta}\left(\left(c_{1}+2 c_{3}+c_{4}+2 c_{6}\right)+\left(d_{1}+d_{4}\right)\right)$ \\
$B^{0} \rightarrow D_{s}^{-} K^{0} \pi^{+}$ & $\frac{\sqrt{2} M_{D_{s}}}{F_{0}} E_{K}\left(\left(c_{3}+c_{4}\right)-\left(d_{3}-d_{4}\right)\right)$ \\
$B^{0} \rightarrow D^{-} \pi^{0} \pi^{+}$ & $-\frac{M_{D}}{F_{0}} E_{\pi}\left(\left(c_{1}+c_{4}\right)+\left(d_{1}-2 d_{3}+d_{4}-2 d_{6}\right)\right)$ \\
$B^{0} \rightarrow \bar{D}^{0} \pi^{-} K^{+}$ & $-s_{1} \frac{\sqrt{2} M_{D}}{F_{0}} E_{\pi}\left(\left(c_{2}+c_{4}\right)+\left(d_{2}+d_{4}\right)\right)$ \\
$B^{0} \rightarrow D^{-} \pi^{0} K^{+}$ & $-s_{1} \frac{M_{D}}{F_{0}} E_{\pi}\left(-\left(c_{4}-c_{6}\right)-\left(d_{4}-d_{6}\right)\right)$ \\
$B^{0} \rightarrow D^{-} \eta K^{+}$ & $-s_{1} \frac{M_{D}}{\sqrt{3} F_{0}} E_{\eta}\left(\left(c_{4}-c_{6}\right)+\left(d_{4}+3 d_{6}\right)\right)$ \\
$B^{0} \rightarrow D_{s}^{-} K^{0} K^{+}$ & $-s_{1} \frac{\sqrt{2} M_{D_{s}}}{F_{0}} E_{K}\left(\left(c_{1}+c_{4}\right)+\left(d_{1}+d_{4}\right)\right)$ \\
$B^{-} \rightarrow D^{0} \pi^{0} K^{-}$ & $-s_{1} \frac{M_{D}}{F_{0}} E_{\pi}\left(\left(c_{1}+c_{4}+c_{2}+c_{6}\right)-\left(d_{1}-d_{2}-d_{4}-d_{6}\right)\right)$ \\
$B^{-} \rightarrow D^{0} \eta K^{-}$ & $-s_{1} \frac{M_{D}}{\sqrt{3} F_{0}} E_{\eta}\left(\left(c_{1}+c_{4}-c_{2}-c_{6}\right)-\left(d_{1}-3 d_{2}-d_{4}-3 d_{6}\right)\right)$ \\
$B^{-} \rightarrow D^{+} \pi^{-} K^{-}$ & $-s_{1} \frac{\sqrt{2} M_{D}}{F_{0}} E_{\pi}\left(\left(c_{1}+c_{4}\right)-\left(d_{1}-d_{4}\right)\right)$ \\
$B^{-} \rightarrow D_{s}^{+} K^{-} K^{+}$ & $-s_{1} \frac{2 \sqrt{2} M_{D_{s}}}{F_{0}} E_{K}\left(c_{1}+c_{4}\right)$ \\
\hline \hline
\end{tabular}

$$
\frac{5}{\sqrt{6}} A:\left(-\frac{1}{\sqrt{3}} A\right):\left(\frac{1}{\sqrt{6}} A+\sqrt{\frac{3}{2}} B\right): A .
$$

Since the $D \pi$ systems with $I=1 / 2$ and $3 / 2$ have both the $D^{0} \pi^{0}$ and the $D^{+} \pi^{-}$components, we need to project out the $D^{+} \pi^{-}$component and obtain the ratio for $D^{+} \pi^{-}(I=1 / 2)$ and $D^{+} \pi^{-}(I=3 / 2)$. As a result, trivial overall factors $\sqrt{\frac{2}{3}}$ and $-\sqrt{\frac{1}{3}}$ are needed for $I=1 / 2$ and $3 / 2$, respectively. Taking into account the chiral symmetry implying that the amplitudes are proportional to the energy of the Goldstone bosons and the FSI, we reobtain the amplitude in Eq. (31).

With the Lagrangian in Eq. (25), one can calculate the production vertices responsible for processes $B_{s}^{0} \rightarrow$ $\bar{D}^{0} K^{-} \pi^{+}, \quad B^{0} \rightarrow \bar{D}^{0} \pi^{-} \pi^{+}, \quad B^{-} \rightarrow D^{+} \pi^{-} K^{-}, \quad$ and $B^{0} \rightarrow$ $\bar{D}^{0} \pi^{-} K^{+}$as well. For these reactions experimental data are available from the LHCb experiment [23,24,56,57]. The weak production vertices needed for those decays are listed in Table II. Note that in Ref. [35], only the terms corresponding to an even relative angular momentum of the two light mesons are considered, i.e., the first term in the Lagrangian (25). It is appropriate for the decay $B^{-} \rightarrow D^{+} \pi^{-} \pi^{-}$and numerically sufficient to describe the decay $B_{s}^{0} \rightarrow \bar{D}^{0} K^{-} \pi^{+}$. We find that the inclusion of the terms for the two light mesons with an odd relative angular momentum does not improve the fit quality significantly, which means that the experimental data used in Ref. [35] are not sufficient to disentangle the contributions from the two terms. In this paper, more experimental data, i.e., $B^{0} \rightarrow \bar{D}^{0} \pi^{-} \pi^{+}, B^{-} \rightarrow D^{+} \pi^{-} K^{-}$, and $B^{0} \rightarrow \bar{D}^{0} \pi^{-} K^{+}$, are considered and a discrimination of both contributions is possible.

Although these decays have three hadrons in the final states, we expect that, at least in the low-energy tails of the invariant mass of the $D \phi$ subsystems, the effects from the crossed-channel FSIs (interactions between the soft and hard light mesons and those between the $D$ meson and the hard pseudoscalar meson) do not produce any nontrivial structure in the $D \phi$ distributions, which is supported by the analyses in Refs. [23,24,56,57]. Therefore, for a description of the low-energy $D \phi$ systems, we do not need to account for the full three-body FSIs. The effects from the crossed-channel FSIs can be simply encoded into an extra undetermined complex factor for each partial wave as it is done in the isobar model. Following the procedures for the $B^{-} \rightarrow D^{+} \pi^{-} \pi^{-}$above, one can obtain the $S$-wave amplitudes as ${ }^{6}$

\footnotetext{
${ }^{6}$ Hereafter, we neglect the overall factor $\frac{\sqrt{2} M_{D}}{F_{0}}$ for the $S$-wave amplitudes by absorbing it into the LECs.
} 


$$
\begin{aligned}
& \mathcal{A}_{0}\left(B_{s}^{0} \rightarrow \bar{D}^{0} K^{-} \pi^{+}\right)=\left(c_{2}+c_{4}+d_{2}+d_{4}\right) E_{K}+d_{6} E_{\pi} G_{\bar{D}_{s} \pi}(s) T_{\bar{D}_{s} \pi \rightarrow \bar{D} \bar{K}}^{1}(s) \\
& +\frac{1}{2}\left(c_{2}-c_{1}+d_{2}-d_{1}\right) E_{K} G_{\bar{D} \bar{K}}(s) T_{\bar{D} \bar{K} \rightarrow \bar{D} \bar{K}}^{1}(s) \\
& +\frac{1}{2}\left(c_{1}+c_{2}+2 c_{4}+d_{1}+d_{2}+2 d_{4}\right) E_{K} G_{\bar{D} \bar{K}}(s) T_{\bar{D} \bar{K} \rightarrow \bar{D} \bar{K}}^{0}(s) \\
& +\sqrt{\frac{1}{3}}\left(c_{4}-c_{6}+d_{4}\right) E_{\eta} G_{\bar{D}_{s} \eta}(s) T_{\bar{D}_{s} \eta \rightarrow \bar{D} \bar{K}}^{0}(s), \\
& \mathcal{A}_{0}\left(B^{0} \rightarrow \bar{D}^{0} \pi^{-} \pi^{+}\right)=\left(c_{2}+c_{3}+c_{4}+2 c_{5}+d_{2}-d_{3}+d_{4}\right) E_{K}+\frac{1}{3} E_{\pi} G_{D \pi}(s) T_{D \pi \rightarrow D \pi}^{1 / 2}(s) \\
& \times\left(c_{1}+2 c_{2}+2 c_{3}+3 c_{4}+4 c_{5}+d_{1}+2 d_{2}-4 d_{3}+3 d_{4}-2 d_{6}\right) \\
& +\frac{1}{3}\left(c_{2}-c_{1}+c_{3}+2 c_{5}-d_{1}+d_{2}+d_{3}+2 d_{6}\right) E_{\pi} G_{D \pi}(s) T_{D \pi \rightarrow D \pi}^{3 / 2}(s) \\
& +\frac{1}{3}\left(c_{1}+2 c_{3}+c_{4}+2 c_{6}+d_{1}+d_{4}\right) E_{\eta} G_{D \eta}(s) T_{D \eta \rightarrow D \pi}^{1 / 2}(s) \\
& +\sqrt{\frac{2}{3}}\left(c_{3}+c_{4}-d_{3}+d_{4}\right) E_{K} G_{D_{s} \bar{K}}(s) T_{D_{s} \bar{K} \rightarrow D \pi}^{1 / 2}(s) \\
& \mathcal{A}_{0}\left(B^{-} \rightarrow D^{+} \pi^{-} K^{-}\right)=-s_{1}\left(c_{1}+c_{4}-d_{1}+d_{4}\right) E_{\pi}-2 \sqrt{\frac{2}{3}} s_{1}\left(c_{1}+c_{4}\right) E_{K} G_{D_{s} \bar{K}}(s) T_{D_{s} \bar{K} \rightarrow D \pi}^{1 / 2}(s) \\
& -s_{1}\left(3 c_{1}+c_{2}+3 c_{4}+c_{6}-3 d_{1}+d_{2}+3 d_{4}+d_{6}\right) E_{\pi} G_{D \pi}(s) T_{D \pi \rightarrow D \pi}^{1 / 2}(s) \\
& -s_{1}\left(c_{1}-c_{2}+c_{4}-c_{6}-d_{1}+d_{4}+3 d_{2}+3 d_{6}\right) E_{\eta} G_{D \eta}(s) T_{D \eta \rightarrow D \pi}^{1 / 2}(s) \\
& +\frac{1}{3} s_{1}\left(c_{2}+c_{6}+d_{2}+d_{6}\right) E_{\pi} G_{D \pi}(s) T_{D \pi \rightarrow D \pi}^{3 / 2}(s) \\
& \mathcal{A}_{0}\left(B^{0} \rightarrow \bar{D}^{0} \pi^{-} K^{+}\right)=-s_{1}\left(c_{2}+c_{4}+d_{2}+d_{4}\right) E_{\pi}-\frac{1}{3} s_{1}\left(2 c_{2}+3 c_{4}-c_{6}+2 d_{2}+3 d_{4}-d_{6}\right) E_{\pi} G_{D \pi}(s) T_{D \pi \rightarrow D \pi}^{1 / 2}(s) \\
& -\frac{1}{3} s_{1}\left(c_{4}-c_{6}+d_{4}+3 d_{6}\right) E_{\eta} G_{D \eta}(s) T_{D \eta \rightarrow D \pi}^{1 / 2}(s) \\
& -\sqrt{\frac{2}{3}} s_{1}\left(c_{1}+c_{4}+d_{1}+d_{4}\right) E_{K} G_{D_{s} \bar{K}}(s) T_{D_{s} \bar{K} \rightarrow D \pi}^{1 / 2}(s) \\
& -\frac{1}{3} s_{1}\left(c_{2}+c_{6}+d_{2}+d_{6}\right) E_{\pi} G_{D \pi} T_{D \pi \rightarrow D \pi}^{3 / 2}(s) .
\end{aligned}
$$

Next, we show that the $S$-wave decay amplitudes above satisfy the two-body unitarity relation

$$
\mathcal{A}-\mathcal{A}^{*}=-2 i T \rho \mathcal{A}^{*}=-2 i T^{\dagger} \rho \mathcal{A},
$$

where $T$ represents the $T$ matrix for the two-body scattering and fulfills the unitarity relation ${ }^{7}$

$$
T-T^{\dagger}=-2 i T^{\dagger} \rho T
$$

which can be rewritten as

$$
\left(T^{\dagger}\right)^{-1}-T^{-1}=-2 i \rho .
$$

\footnotetext{
${ }^{7}$ Here, $T$ is defined via $S=1-i T$, with $S$ the $S$-matrix.
}

The $T$ matrix in Ref. [30] is given by

$$
T=V[1-\tilde{G} V]^{-1}=\left[V^{-1}-\tilde{G}\right]^{-1},
$$

where $V$ is the $S$-wave projection of the $D \phi$ scattering potentials from ChPT and $\tilde{G}$ is a diagonal matrix whose nonvanishing elements are the loop functions with the same form as the $G(s)$ function in Eq. (29) [30]. It can be rewritten in a more concise form

$$
T^{-1}=V^{-1}-\tilde{G}
$$

Since the $\tilde{G}$ matrix elements satisfy $\operatorname{Im} \tilde{G}_{i i}(s)=-\rho_{i i}(s)$, with $\rho_{i i}(s)$ the two-body phase space factor in the $i$ th channel, and $V(s)$ is real for real $s$, it is easy to conclude 
that the $T$ matrix fulfills the two-body unitarity in Eqs. (45) and (46).

To show that the decay amplitudes in Eqs. (31) and (40)-(43) fulfill the two-body unitarity in Eq. (44), we write them in a matrix form

$$
\mathcal{A}=P+T G P .
$$

In components, this reads

$$
\mathcal{A}_{i}=P_{i}+\sum_{j} P_{j} G_{j} T_{j i}
$$

where $P_{i}$ represents the weak production vertices given in Table II. Making use of Eq. (48), the decay amplitudes in Eq. (49) can be rewritten as

$$
\mathcal{A}=(1+T G) P=T\left(T^{-1}+G\right) P=T\left(V^{-1}+G-\tilde{G}\right) P .
$$

Because of the time reversal symmetry of the strong interaction, which means that $T^{\dagger}=T^{*}$, one obtains the desired two-body unitarity for the decay amplitudes

$$
\begin{aligned}
-2 i T^{\dagger} \rho \mathcal{A} & =-2 i T^{\dagger} \rho T\left(V^{-1}+G-\tilde{G}\right) P \\
& =\left(T-T^{*}\right)\left(V^{-1}+G-\tilde{G}\right) P \\
& =\mathcal{A}-\mathcal{A}^{*} .
\end{aligned}
$$

Having constructed the $S$-wave amplitudes, the complex decay amplitudes for the $P$ and $D$ waves are described by an isobar model as coherent sums of intermediate resonant decays. This is reasonable because of the relatively narrow widths of the resonances in $P$ and $D$ waves. The $P$ - and $D$-wave amplitudes used in Ref. [35] have the same BW form as that in the LHCb analysis [38]. For the lowenergy $D^{+} \pi^{-}$systems in processes $B^{-} \rightarrow D^{+} \pi^{-} \pi^{-}$and $B^{-} \rightarrow D^{+} \pi^{-} K^{-}$, the $P$ - and $D$-wave resonant contributions considered here include the ground-state charmed vector meson $D^{*}$ and an excited state $D_{1}^{*}(2680)$ [38] in the $P$ wave, ${ }^{8}$ and the $D_{2}^{*}(2460)$ in the $D$ wave as in Ref. [35]. For these decays, the $P$ - and $D$-wave amplitudes are given by

$$
\begin{aligned}
& \mathcal{A}_{1}\left(B^{-} \rightarrow D^{+} \pi^{-} M\right)=c_{D^{*}} F_{D^{*}}(s, z)+c_{D^{*}}^{\prime} F_{D_{1}^{*}(2680)}(s, z), \\
& \mathcal{A}_{2}\left(B^{-} \rightarrow D^{+} \pi^{-} M\right)=c_{D_{2}} F_{D_{2}}(s, z),
\end{aligned}
$$

\footnotetext{
${ }^{8}$ The width of the $D_{1}^{*}(2680)$ is large and a BW parametrization is questionable in principle. However, we are only interested in the energy region up to $M_{D \pi}=2.54 \mathrm{GeV}$ as in Ref. [35] with a particular focus on the $S$ wave. The $D_{1}^{*}(2680)$, whose mass is about $140 \mathrm{MeV}$ (similar to the value of its width) higher than the upper edge of the fit window, plays a role of providing a $P$-wave background which interferes with other partial waves in the angular moments. Since in this region the $P$-wave phase is smooth, a BW parametrization for the $D_{1}^{*}(2680)$ should be sufficient.
}

where the complex coefficients $c_{D^{*}}, c_{D^{*}}^{\prime}$, and $c_{D_{2}}$ vary for different decays and will be determined by fitting to the experimental data. $F_{i}(s, z)$ represents the contribution of each resonance to the corresponding decay. For the decays $B^{0} \rightarrow \bar{D}^{0} \pi^{-} \pi^{+}$and $B^{0} \rightarrow \bar{D}^{0} \pi^{-} K^{+}$, the vector and tensor charmed mesons $D^{*}$ and $D_{2}^{*}(2460)$ are taken into account as those in the LHCb analysis [23,24],

$$
\begin{aligned}
& \mathcal{A}_{1}\left(B^{0} \rightarrow \bar{D}^{0} \pi^{-} M\right)=c_{D^{*}} F_{D^{*}}(s, z), \\
& \mathcal{A}_{2}\left(B^{0} \rightarrow \bar{D}^{0} \pi^{-} M\right)=c_{D_{2}} F_{D_{2}}(s, z) .
\end{aligned}
$$

For the decay $B_{s}^{0} \rightarrow \bar{D}^{0} K^{-} \pi^{+}$, we are interested in the low-energy region of the $\bar{D}^{0} K^{-}$. The strange partners of the ones in Eq. (54), i.e., the $D_{s}^{*}$ in the $P$ wave and $D_{s 2}(2573)$ in the $D$ wave are included as

$$
\begin{aligned}
& \mathcal{A}_{1}\left(B_{s}^{0} \rightarrow \bar{D}^{0} K^{-} \pi^{+}\right)=c_{D_{s}^{*}} F_{D_{s}^{*}}(s, z), \\
& \mathcal{A}_{2}\left(B_{s}^{0} \rightarrow \bar{D}^{0} K^{-} \pi^{+}\right)=c_{D_{s 2}} F_{D_{s 2}}(s, z) .
\end{aligned}
$$

$F_{i}(s, z)$ is a function of both the $D \phi$ invariant mass and $z$, the cosine of the $D \phi$ helicity angle. It has the form

$$
\begin{aligned}
F^{(J)}(s, z)= & R^{(J)}(s) \times X^{(J)}\left(|\vec{p}| r_{\mathrm{BW}}\right) \\
& \times X^{(J)}\left(|\vec{q}| r_{\mathrm{BW}}\right) \times M^{(J)}(\vec{p}, \vec{q}),
\end{aligned}
$$

where $\vec{p}$ and $\vec{q}$ are the momenta of the hard light meson $M$ and the one of the resonance daughters, respectively, both evaluated in the rest frame of the resonance. Here, the relativistic $\mathrm{BW}$ propagator is given by

$$
\begin{aligned}
R^{(J)}\left(m^{2}\right) & =\frac{1}{\left(m_{0}^{2}-m^{2}\right)-i m_{0} \Gamma^{(J)}(m)}, \\
\Gamma^{(J)}(m) & =\Gamma_{0}\left(\frac{q}{q_{0}}\right)^{2 J+1}\left(\frac{m_{0}}{m}\right) X^{(J) 2}\left(q r_{\mathrm{BW}}\right),
\end{aligned}
$$

where $q=|\vec{q}|, \Gamma_{0}$ is the width of the static resonance, and $q_{0}$ is $q$ evaluated at the resonance mass $m_{0}$. The $P$ - and $D$-wave Blatt-Weisskopf barrier factors $X^{(J)}(x)$ are given by

$$
\begin{aligned}
& X^{(1)}(x)=\sqrt{\frac{1+x_{0}^{2}}{1+x^{2}}}, \\
& X^{(2)}(x)=\sqrt{\frac{x_{0}^{4}+3 x_{0}^{2}+9}{x^{4}+3 x^{2}+9}},
\end{aligned}
$$

where $x_{0}$ represents the value of $x$ evaluated at $m=m_{0}$. The radius of the barrier, $r_{\mathrm{BW}}$, is taken to be $4.0 \mathrm{GeV}^{-1}$ $[23,24,38,56,57]$. The angular probability distribution terms, $M^{(J)}$, are given in the Zemach tensor formalism [60] by

$$
\begin{aligned}
& M^{(1)}(\vec{p}, \vec{q})=-2 \vec{p} \cdot \vec{q}, \\
& M^{(2)}(\vec{p}, \vec{q})=\frac{4}{3}\left(3(\vec{p} \cdot \vec{q})^{2}-(|\vec{p}||\vec{q}|)^{2}\right),
\end{aligned}
$$


which have similar structures as the Legendre polynomials referring to the angle between $\vec{p}$ and $\vec{q}$. The virtual contributions from the resonances with masses outside the kinematically allowed region, e.g., $D^{*}$, are described by the function $F_{i}(s, t)$ as well, however, with the modification that the mass $m_{0}$ is replaced by an effective mass $m_{0}^{\text {eff }}$ which is given by (see, e.g., Ref. [38])

$m_{0}^{\mathrm{eff}}=m^{\min }+\left(m^{\mathrm{max}}-m^{\min }\right)\left(1+\tanh \frac{m_{0}-\frac{m^{\min }+m^{\max }}{2}}{m^{\max }-m^{\min }}\right)$,

where $m^{\max }$ and $m^{\min }$ represent the upper and lower limits of the kinematically allowed mass range.

\section{NUMERICAL ANALYSIS}

In this section, we demonstrate that the amplitudes in Eqs. (40)-(43) can indeed describe the data collected at the LHCb experiments in Refs. [23,24,56,57]. We fit to the socalled angular moments, which contain important information about the partial-wave phase variations. The angular moments $\left\langle P_{L}\right\rangle$ are obtained by weighting the event distribution in the invariant mass by the Legendre polynomial of order $L$ with respect to $z$,

$$
\left\langle P_{L}(s)\right\rangle=\int_{-1}^{1} d z \frac{d \Gamma}{d \sqrt{s} d z} P_{L}(z)
$$

The angular moments are most powerful when a resonance is present only in one invariant mass combination. The structures show up in moments up to $2 J$, where $J$ is the spin of the contributing resonance [56]. Neglecting partial waves with $L \geq 3$, the first few unnormalized moments (normalized relative to each another) are given by

$$
\begin{aligned}
\left\langle P_{0}\right\rangle & \propto\left|\mathcal{A}_{0}\right|^{2}+\left|\mathcal{A}_{1}\right|^{2}+\left|\mathcal{A}_{2}\right|^{2}, \\
\left\langle P_{1}\right\rangle \propto & \frac{2}{\sqrt{3}}\left|\mathcal{A}_{0}\right|\left|\mathcal{A}_{1}\right| \cos \left(\delta_{0}-\delta_{1}\right) \\
& +\frac{4}{\sqrt{15}}\left|\mathcal{A}_{1}\right|\left|\mathcal{A}_{2}\right| \cos \left(\delta_{1}-\delta_{2}\right), \\
\left\langle P_{2}\right\rangle \propto & \frac{2}{5}\left|\mathcal{A}_{1}\right|^{2}+\frac{2}{7}\left|\mathcal{A}_{2}\right|^{2}+\frac{2}{\sqrt{5}}\left|\mathcal{A}_{0}\right|\left|\mathcal{A}_{2}\right| \cos \left(\delta_{0}-\delta_{2}\right), \\
\left\langle P_{3}\right\rangle \propto & \frac{6}{7} \sqrt{\frac{3}{5}}\left|\mathcal{A}_{1}\right|\left|\mathcal{A}_{2}\right| \cos \left(\delta_{1}-\delta_{2}\right),
\end{aligned}
$$

where $\delta_{i}$ is the phase of $\mathcal{A}_{i}$, i.e., $\mathcal{A}_{i}=\left|\mathcal{A}_{i}\right| e^{i \delta_{i}}$. Instead of $\left\langle P_{1}\right\rangle$ and $\left\langle P_{3}\right\rangle$, we analyze their linear combination as proposed in Ref. [35],

$$
\left\langle P_{13}\right\rangle=\left\langle P_{1}\right\rangle-\frac{14}{9}\left\langle P_{3}\right\rangle \propto \frac{2}{\sqrt{3}}\left|\mathcal{A}_{0}\right|\left|\mathcal{A}_{1}\right| \cos \left(\delta_{0}-\delta_{1}\right),
$$

TABLE III. The masses and widths of the $P$ - and $D$-wave resonances are taken from the LHCb analyses $[38,56]$.

\begin{tabular}{lccc}
\hline \hline Resonance & Spin & Mass $(\mathrm{MeV})$ & Width $(\mathrm{MeV})$ \\
\hline$D^{*}$ & 1 & 2006.98 & 2.1 \\
$D_{2}^{*}(2460)$ & 2 & 2463.7 & 47.0 \\
$D_{1}^{*}(2680)$ & 1 & 2681.1 & 186.7 \\
$D_{s}^{*}$ & 1 & 2112.1 & 1.9 \\
$D_{s 2}(2573)$ & 2 & 2568.39 & 16.9 \\
\hline \hline
\end{tabular}

which only depends on the $S$-P interference up to $L=2$ and is particularly sensitive to the $S$-wave phase motion.

Before fitting to the experimental data of the angular moments, let us investigate the $S$-wave amplitudes in Eqs. (40)-(43) in more detail. Although there are 11 unknown LECs in the Lagrangian (25), only ten independent combinations are involved in this work. Furthermore, to reduce their correlations in the fit procedure, the LECs are redefined as

$$
\begin{aligned}
A=c_{1}+c_{4}, & B=\frac{3}{2}\left(c_{2}+c_{6}\right), & \\
C=c_{2}+c_{4}, & D=c_{3}+2 c_{5}, & E=c_{3}+c_{6}, \\
A^{\prime}=d_{1}-d_{4}, & B^{\prime}=d_{2}+d_{6}, & \\
C^{\prime}=d_{4}-d_{6}, & D^{\prime}=d_{4}+d_{6}, & E^{\prime}=d_{3} .
\end{aligned}
$$

In particular, the definitions of $A, B$ are the same as that in Ref. [35], and thus we will take the ratio $B / A$ determined therein by fitting to the high-quality data for the decay $B^{-} \rightarrow D^{+} \pi^{-} \pi^{-}$, where $B / A$ and the subtraction constant in the $G$ function are the only free parameters for the $S$-wave amplitude. ${ }^{9}$ Moreover, although the subtraction constants for different channels could be different in principle, as in Ref. [35], a uniform value is taken to reduce the number of free parameters. For the $P$ - and $D$-wave amplitudes in Eqs. (53)-(55), two real parameters, the magnitude and the phase of the corresponding $c_{D}$, are needed for each resonance. Since we are not interested in the precise $P$ - and $D$-wave amplitudes, the resonance mass and width are taken as the central values reported in the $\mathrm{LHCb}$ analyses $[38,56]$ and the uncertainties therein are not considered. The used resonance parameters are listed in Table III.

Moreover, we notice that only three sets of the weak production vertices in Table II are independent. Thus instead of fitting the four decay amplitudes to the experimental angular moments simultaneously, we fix the LECs in the amplitudes in Eqs. (40)-(43) by fitting to three of them, i.e., $B^{-} \rightarrow D^{+} \pi^{-} K^{-}, B_{s}^{0} \rightarrow \bar{D}^{0} K^{-} \pi^{+}$, and $B^{0} \rightarrow$ $\bar{D}^{0} \pi^{-} \pi^{+}$, and then describe the angular moments for $B^{0} \rightarrow$ $\bar{D}^{0} \pi^{-} K^{+}$with the determined LECs. We fit to the data of

\footnotetext{
${ }^{9}$ The value of $A$ cannot be determined since it serves as the normalization constant for the $S$-wave contribution as well.
} 

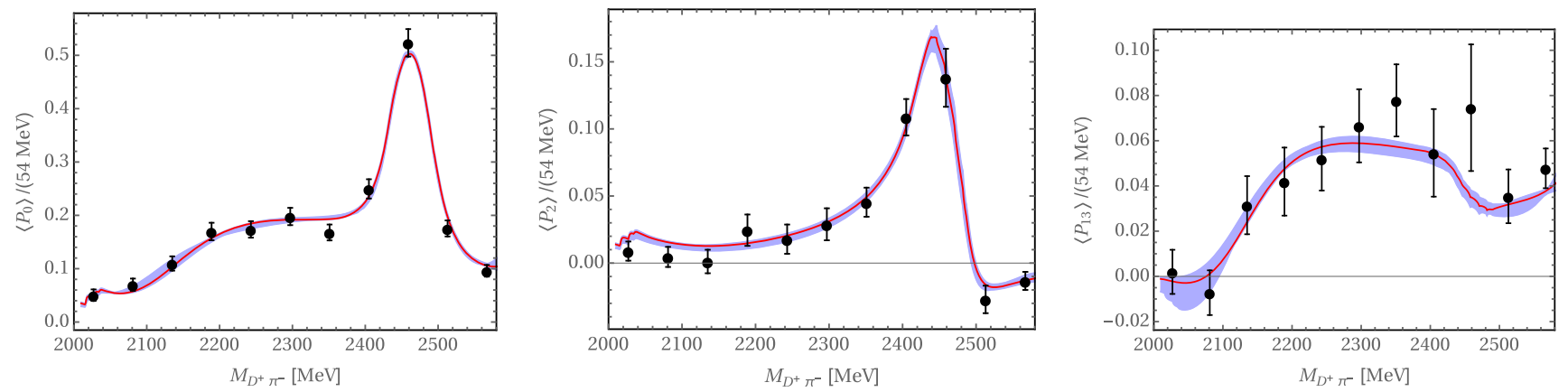

FIG. 3. Fit to the LHCb data of the angular moments $\left\langle P_{0}\right\rangle,\left\langle P_{2}\right\rangle$, and $\left\langle P_{13}\right\rangle$ for the $B^{-} \rightarrow D^{+} \pi^{-} K^{-}$reaction [57]. The largest error of $\left\langle P_{1}\right\rangle$ and $14\left\langle P_{3}\right\rangle / 9$ in each bin is taken as the error of $\left\langle P_{13}\right\rangle$. The solid lines show our best fit results with error bands corresponding to the one-sigma uncertainties propagated from the input scattering amplitudes.
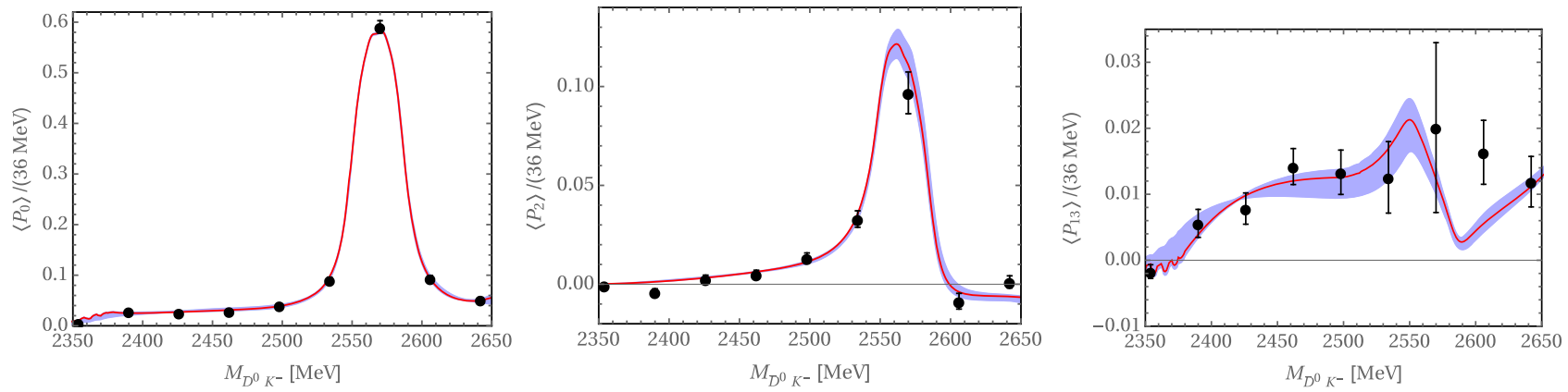

FIG. 4. Fit to the LHCb data of the angular moments $\left\langle P_{0}\right\rangle,\left\langle P_{2}\right\rangle$, and $\left\langle P_{13}\right\rangle$ for the $B_{s}^{0} \rightarrow \bar{D}^{0} K^{-} \pi^{+}$reaction [56]. The largest error of $\left\langle P_{1}\right\rangle$ and $14\left\langle P_{3}\right\rangle / 9$ in each bin is taken as the error of $\left\langle P_{13}\right\rangle$. The error bands correspond to the one-sigma uncertainties propagated from the input scattering amplitudes.

the moments $\left\langle P_{0}\right\rangle,\left\langle P_{13}\right\rangle$, and $\left\langle P_{2}\right\rangle$ defined in Eqs. (62) and (63) up to $M_{D \pi}=2.54 \mathrm{GeV}$ as in Ref. [35] for the decays $B^{-} \rightarrow D^{+} \pi^{-} K^{-}$and $B^{0} \rightarrow \bar{D}^{0} \pi^{-} \pi^{+}$, and up to $M_{\bar{D} \bar{K}}=$ $2.65 \mathrm{GeV}$ for $B_{s}^{0} \rightarrow \bar{D}^{0} K^{-} \pi^{+}$. The best fit results to the $\mathrm{LHCb}$ data of the angular moments for the reactions $B^{-} \rightarrow D^{+} \pi^{-} K^{-}, B_{s}^{0} \rightarrow \bar{D}^{0} K^{-} \pi^{+}$, and $B^{0} \rightarrow \bar{D}^{0} \pi^{-} \pi^{+}$are shown in Figs. 3-5, respectively. The best fit has a reasonable quality with $\chi^{2} /$ d.o.f. $=1.2$ and the parameter values are listed in Tables IV and V. The bands in Figs. 3-5 reflect the one-sigma errors of the parameters in the scattering amplitudes determined in Ref. [30].

It is worth mentioning that in $\left\langle P_{13}\right\rangle$ for the reaction $B^{-} \rightarrow D^{+} \pi^{-} K^{-}$, where the $D_{2}(2460)$ does not play any role, the data show significant variations between 2.4 and $2.5 \mathrm{GeV}$, as that for $B^{-} \rightarrow D^{+} \pi^{-} \pi^{-}$in Ref. [35]. The data for the $B^{0} \rightarrow \bar{D}^{0} \pi^{-} \pi^{+}$and $B^{0} \rightarrow \bar{D}^{0} \pi^{-} K^{+}$also have a similar behavior, but with lower statistics; see Figs. 5 and 9. These features are in line with the expectation due to the
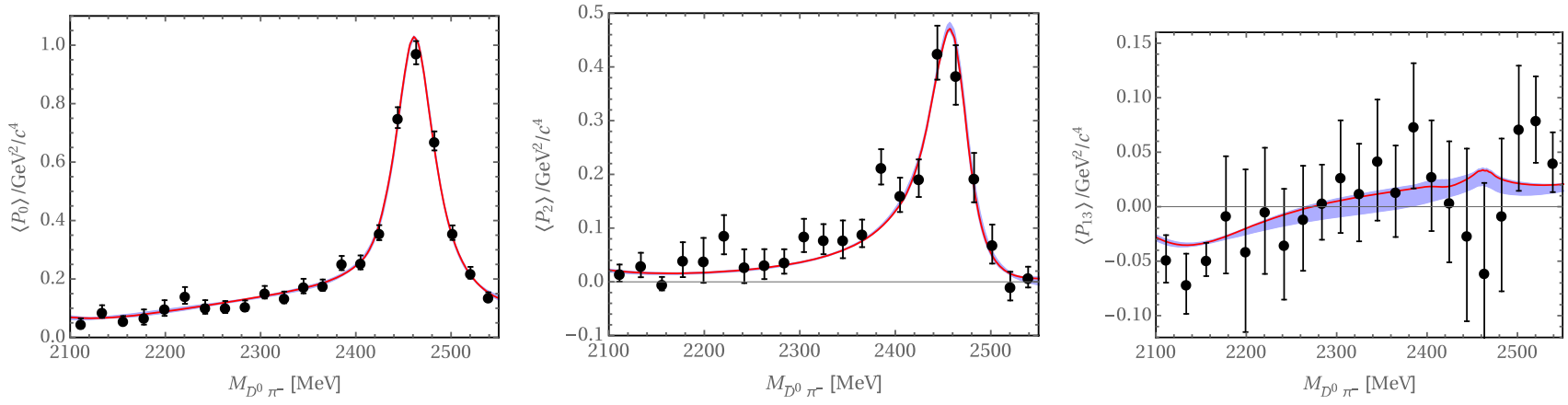

FIG. 5. Fit to the LHCb data of the angular moments $\left\langle P_{0}\right\rangle,\left\langle P_{13}\right\rangle$, and $\left\langle P_{2}\right\rangle$ for the $B^{0} \rightarrow \bar{D}^{0} \pi^{-} \pi^{+}$reaction [24]. The largest error of $\left\langle P_{1}\right\rangle$ and $14\left\langle P_{3}\right\rangle / 9$ in each bin is taken as the error of $\left\langle P_{13}\right\rangle$. The error bands correspond to the one-sigma uncertainties propagated from the input scattering amplitudes. 
TABLE IV. Values of the LECs from fitting to the LHCb data of the angular moments $\left\langle P_{0}\right\rangle,\left\langle P_{2}\right\rangle$, and $\left\langle P_{13}\right\rangle$ for the reactions $B^{-} \rightarrow D^{+} \pi^{-} K^{-}, B_{s}^{0} \rightarrow \bar{D}^{0} K^{-} \pi^{+}$, and $B^{0} \rightarrow \bar{D}^{0} \pi^{-} \pi^{+}$[24,56,57]. The two errors correspond to the one-sigma uncertainties propagated from the input scattering amplitudes and from fitting to the experimental data, respectively. The asterisk marks an input value from Ref. [35].

\begin{tabular}{lcccc}
\hline \hline$B / A^{*}$ & $C / A$ & $D / A$ & $E / A$ & $A^{\prime} / A$ \\
\hline$-3.6 \pm 0.4 \pm 0.1$ & $-2.3_{-0.5-0.7}^{+1.3+0.5}$ & $2.0_{-1.0-1.1}^{+2.7+1.1}$ & $-1.6_{-0.3-1.2}^{+1.0+1.2}$ & $4.2_{-2.2-0.8}^{+0.9+1.0}$ \\
\hline \hline$B^{\prime} / A$ & $C^{\prime} / A$ & $D^{\prime} / A$ & $E^{\prime} / A$ \\
\hline $2.4_{-6.5-1.3}^{+1.3+1.3}$ & $1.4_{-2.2-1.7}^{+7.0+1.7}$ & $-3.4_{-0.5-0.3}^{+0.8+0.3}$ & $2.4_{-1.0-1.5}^{+3.9+1.1}$ \\
\hline \hline
\end{tabular}

TABLE V. Values of the subtraction constant $a_{A}$ and the phase parameters denoted by $\delta_{D^{*}}, \delta_{D^{*}}^{\prime}$, and $\delta_{D_{2}}$. The two errors correspond to the uncertainties propagated from the input scattering amplitudes and experimental data.

\begin{tabular}{ccccc}
\hline \hline & $a_{A}$ & $\delta_{D^{*}}$ & $\delta_{D^{*}}^{\prime}$ & $\delta_{D_{2}}$ \\
\hline$B^{-} \rightarrow D^{+} \pi^{-} K^{-}$ & $0.4_{-0.2-0.1}^{+0.1+0.2}$ & $-1.2_{-0.5-0.3}^{+0.1+0.2}$ & $-0.9_{-0.2-0.7}^{+0.9+0.9}$ & $-1.2_{-0.9-0.4}^{+0.2+0.4}$ \\
$B_{s}^{0} \rightarrow \bar{D}^{0} K^{-} \pi^{+}$ & $-1.2_{-0.2-0.0}^{+0.0+0.1}$ & $-2.9_{-0.0-0.1}^{+0.4+0.1}$ & $\cdots$ & $-3.1_{-0.1-0.1}^{+0.5+0.1}$ \\
$B^{0} \rightarrow \bar{D}^{0} \pi^{-} \pi^{+}$ & $-0.1_{-0.4-0.2}^{+0.1+0.1}-0.3_{-0.3-0.3}^{+0.3+0.3}$ & $\cdots$ & $-2.3_{-0.2-0.3}^{+0.3+0.3}$ \\
\hline \hline
\end{tabular}

opening of the $D \eta$ and $D_{s} \bar{K}$ thresholds, respectively, which leads to two cusps in the amplitude. This also implies the importance of the coupled-channel effects. Moreover, using the parameters in Tables IV and V, we can predict the moments $\left\langle P_{1}\right\rangle$ and $\left\langle P_{3}\right\rangle$ separately, and the predictions are fully in line with the experimental data [24,56,57]; see Figs. 6-8.

With the determined LECs in Table IV, we proceed to compare the angular moments for $B^{0} \rightarrow \bar{D}^{0} \pi^{-} K^{+}$obtained from the amplitudes in Eqs. (43) and (54) with the LHCb data [23]. Except for the three normalization parameters (two for the resonances and one for the $S$ wave), there are only three real free parameters: two phase factors for the resonances and one subtraction constant $a_{A}$ in the $G$ function in the $S$-wave amplitude. We perform a fit in the energy region $[2.10,2.54] \mathrm{GeV}$ and the fit result is shown in Fig. 9. The best fit has a $\chi^{2} /$ d.o.f. $=1.8$ and the parameter values are $a_{A}=0.4_{-0.5}^{+0.8}, \delta_{D^{*}}=-2.5_{-0.2}^{+0.2}$, and $\delta_{D_{2}}=-5.9_{-0.2}^{+0.2}$, where only the errors from fitting to the experimental data are given. From Figs. 3-9, we see that the amplitudes in Eqs. (40)-(43) indeed can describe the experimental data collected by the $\mathrm{LHCb}$ Collaboration $[23,24,56,57]$ quite well. Since the poles in those scattering amplitudes in the complex energy plane correspond to the
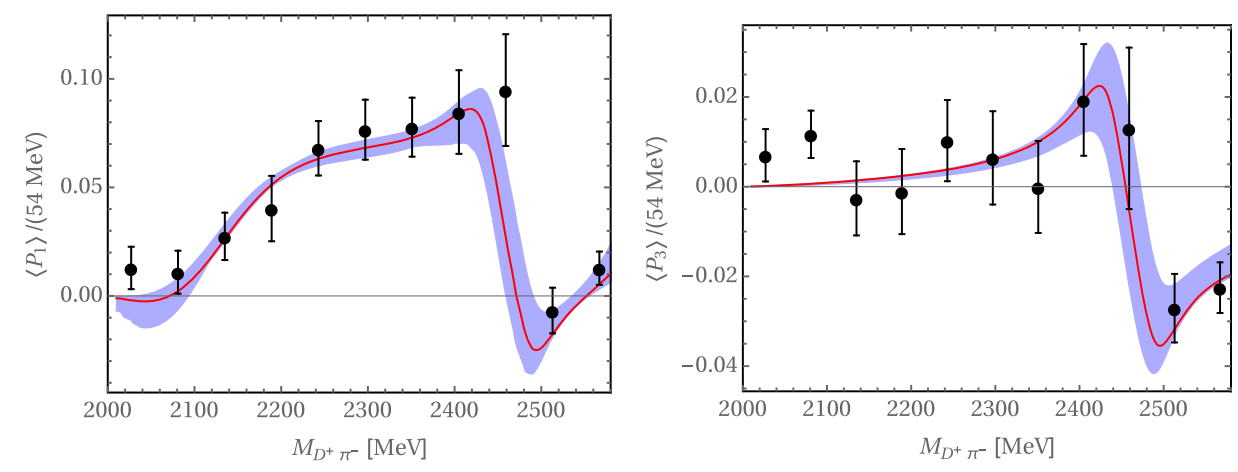

FIG. 6. Comparison of the predictions with the LHCb data of the angular moments $\left\langle P_{1}\right\rangle$ and $\left\langle P_{3}\right\rangle$ for the reaction $B^{-} \rightarrow D^{+} \pi^{-} K^{-}$ [57]. The error bands correspond to the one-sigma uncertainties propagated from the input scattering amplitudes.
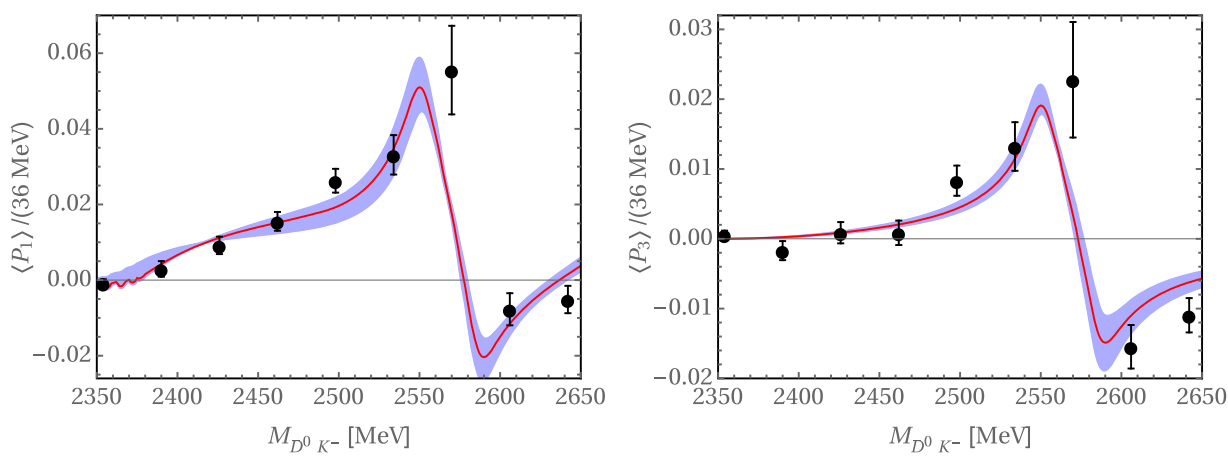

FIG. 7. Comparison of the predictions with the LHCb data of the angular moments $\left\langle P_{1}\right\rangle$ and $\left\langle P_{3}\right\rangle$ for the reaction $B_{s}^{0} \rightarrow \bar{D}^{0} K^{-} \pi^{+}[56]$. The error bands correspond to the one-sigma uncertainties propagated from the input scattering amplitudes. 

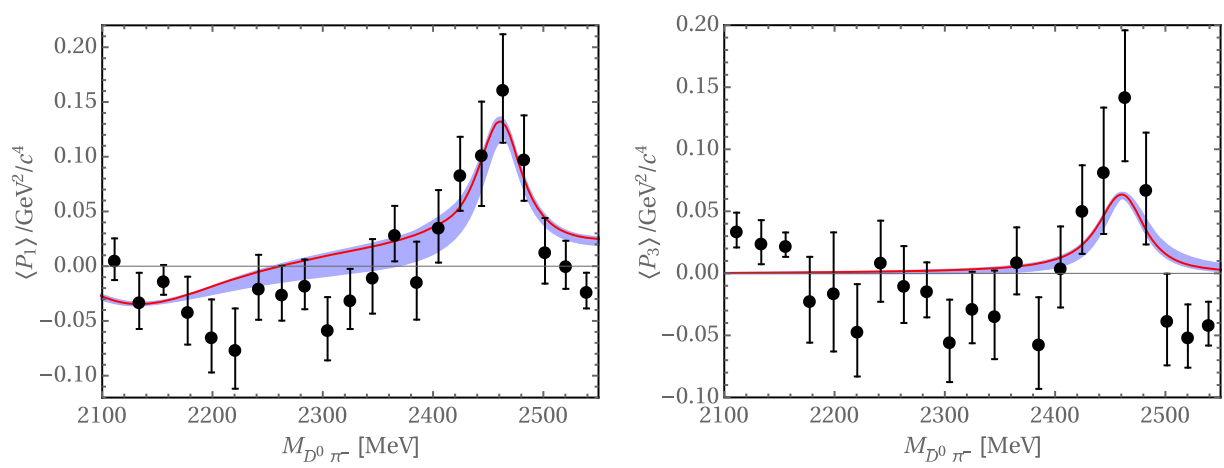

FIG. 8. Comparison of the predictions with the LHCb data for the angular moments $\left\langle P_{1}\right\rangle$ and $\left\langle P_{3}\right\rangle$ for the reaction $B^{0} \rightarrow \bar{D}^{0} \pi^{-} \pi^{+}$[24]. The error bands correspond to the one-sigma uncertainties propagated from the input scattering amplitudes.
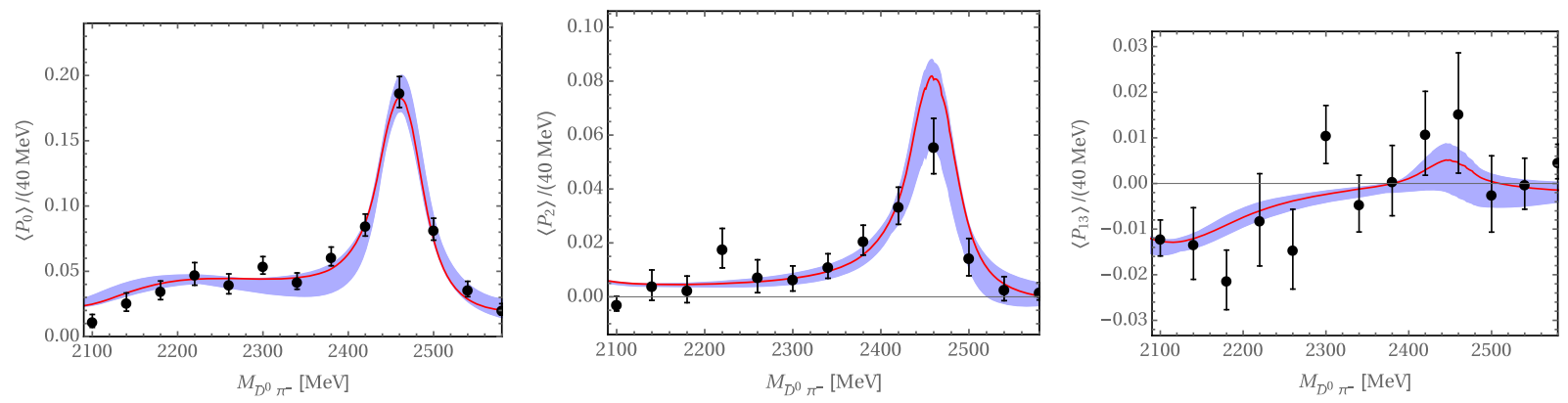

FIG. 9. Fit to the LHCb data of the angular moments for the $B^{0} \rightarrow \bar{D}^{0} \pi^{-} K^{+}$reaction [23]. The shape of the $S$ wave is determined by only one real parameter $a_{A}$. The error bands correspond to the one-sigma uncertainties propagated from the input scattering amplitudes.

scalar charmed meson resonances, one can conclude that the scalar charmed meson spectrum predicted in Refs. [35,37] is consistent with the LHCb data which are the best available data for the scalar charmed mesons. In particular, two poles corresponding to two different resonances exist in the $I=1 / 2$ nonstrange channel. The analyses in this work and in Ref. [35] provide strong support to such a spectrum, and thus the broad $D_{0}^{*}(2400)$ listed in the RPP [21] should be replaced by two states as advocated in Refs. [35,37]. The lower one has a mass of around $2.1 \mathrm{GeV}$ and is much lighter than that extracted from experiments using a naive single-channel BW parametrization.

\section{SUMMARY}

We have shown that the constraints from chiral symmetry are not fulfilled by the standard BW parametrization for a pionic resonance. Using the BW parametrization to fit to the data can lead to a mass larger than its real value. In the case where the resonance width $\Gamma$ is relatively narrow, one can show that the induced shift is proportional to $\Gamma^{2}$. Nevertheless, a naive modification of the BW parametrization by introducing a pion energy factor is neither practical nor systematic since it can only be applied in a very limited energy region before the coupled-channel effects become sizable. Fortunately, unitarized ChPT provides a framework with the chiral symmetry constraint built in and coupledchannel effects taken into account. In the energy regime not far from the threshold of a charmed meson and a light pseudoscalar meson, it can thus be used to analyze the nonleptonic $B$-meson three-body decays to charmed mesons with the emission of two light pseudoscalar mesons which provide access to the scalar charmed meson spectrum.

In order to consider in detail the FSIs between the charmed meson and the light pseudoscalar meson for the reactions $B^{-} \rightarrow D^{+} \pi^{-} \pi^{-}, B_{s}^{0} \rightarrow \bar{D}^{0} K^{-} \pi^{+}, B^{0} \rightarrow \bar{D}^{0} \pi^{-} \pi^{+}$, $B^{-} \rightarrow D^{+} \pi^{-} K^{-}$, and $B^{0} \rightarrow \bar{D}^{0} \pi^{-} K^{+}$, we have constructed a chiral effective Lagrangian for the decays of the $B$ and $B_{s}$ mesons into a charmed meson associated with the emission of two light pseudoscalar mesons. Considering two-body coupled-channel unitarity, we have constructed the $D \phi$ $S$-wave amplitudes for these decays by making use of the effective weak Lagrangian. The involved LECs are thus free parameters in this framework. We have performed fits to the angular moments reported by the $\mathrm{LHCb}$ Collaboration for a series of related decays $B_{s}^{0} \rightarrow$ $\bar{D}^{0} K^{-} \pi^{+}, \quad B^{0} \rightarrow \bar{D}^{0} \pi^{-} \pi^{+}, \quad B^{-} \rightarrow D^{+} \pi^{-} K^{-}$, and $B^{0} \rightarrow$ $\bar{D}^{0} \pi^{-} K^{+}[23,24,56,57]$. In particular, the linear combination of two angular moments $\left\langle P_{13}\right\rangle=\left\langle P_{1}\right\rangle-14\left\langle P_{3}\right\rangle / 9$ only depends on the $S-P$ interference when partial waves with $L \geq 3$ are neglected. Thus, $\left\langle P_{13}\right\rangle$ is the quantity that one should focus on in order to learn about the scalar 
charmed mesons. We have found that the LHCb angular moment data for all these decays can be well described. The predicted $\left\langle P_{1}\right\rangle$ and $\left\langle P_{3}\right\rangle$ also agree with the measurements. Because the FSIs in these fits are taken from the unitarized ChPT amplitudes that were already fixed in Ref. [30], our analysis has implied that the poles contained in these amplitudes can be regarded as the low-lying scalar charmed meson spectrum, and such a spectrum is consistent with the best data $[23,24,38,56,57]$ in that sector. In particular, the poles of the scalar charm-nonstrange mesons, $\left(2105_{-8}^{+6}-i 102_{-11}^{+10}\right) \mathrm{MeV}$ and $\left(2451_{-26}^{+35}-i 134_{-8}^{+7}\right) \mathrm{MeV}$ [35], are different from the resonance parameters of the $D_{0}^{*}(2400)$ listed in Ref. [21], which were extracted using a simple BW parametrization. The analysis in this work gives further strong support to the two- $D_{0}^{*}$ scenario as advocated in Refs. [35,37].

The scenario needs to be confronted with more precise measurements of the angular moments; in particular we urge experimentalists to report directly their measurements of the linear combination $\left\langle P_{13}\right\rangle$, which is very sensitive to the $S$-wave phase motion. Heavy-quark spin symmetry relates the scalar (pseudoscalar) charmed mesons to the axial-vector (vector) ones. Replacing the $D$ meson in the final states by the $D^{*}$, we expect that the $\left\langle P_{13}\right\rangle$ combination for these three-body $\bar{B} \rightarrow D^{*} \pi \pi / K$ decays will be sensitive to the two- $D_{1}$ structure (for more discussion, see Ref. [35]). Moreover, since the higher $D_{0}^{*}\left(D_{1}\right)$ state couples more strongly to the $D_{s}^{(*)} \bar{K}$, this may lead to near-threshold enhancements in the $D_{s}^{(*)} \bar{K}$ spectrum in processes such as
$B^{-} \rightarrow D_{s}^{(*)+} K^{-} \pi^{-}, B^{-} \rightarrow D_{s}^{(*)+} K^{-} K^{-}$, and $\bar{B}_{s}^{0} \rightarrow D_{s}^{(*)+} \bar{K}^{0} \pi^{-}$. In fact, clear near-threshold enhancements can be seen from the $B A B A R$ and Belle measurements of the $D_{s} \bar{K}$ invariant mass distribution for the process $B^{-} \rightarrow D_{s}^{+} K^{-} \pi^{-}[61,62]$ (see also $B^{0} \rightarrow D_{s}^{-} K_{S} \pi^{+}$by Belle [63]). More precise measurements for these processes with an analysis of the angular moments will be very valuable for the understanding of the positive-parity charmed mesons.

Better knowledge of the scalar and axial-vector charmed mesons as well as the corresponding bottom counterparts are expected with the Belle-II [64] and the upcoming highluminosity and high-energy LHC [65].

\section{ACKNOWLEDGMENTS}

This work is supported in part by the Deutsche Forschungsgemeinschaft (DFG) and the National Natural Science Foundation of China (NSFC) through the funds provided to the Sino-German Collaborative Research Center "Symmetries and the Emergence of Structure in QCD" (NSFC Grant No. 11621131001 and DFG Grant No. TRR110), by the NSFC under Grants No. 11747601 and No. 11835015, by the Chinese Academy of Sciences (CAS) under Grants No. QYZDB-SSW-SYS013 and No. XDPB09, by the CAS Center for Excellence in Particle Physics, by the CAS President's International Fellowship Initiative (Grant No. 2018DM0034), and by the VolkswagenStiftung (Grant No. 93562).
[1] B. Aubert et al. (BABAR Collaboration), Phys. Rev. Lett. 90, 242001 (2003).

[2] D. Besson et al. (CLEO Collaboration), Phys. Rev. D 68, 032002 (2003); 75, 119908(E) (2007).

[3] S. Godfrey and N. Isgur, Phys. Rev. D 32, 189 (1985).

[4] S. Godfrey and K. Moats, Phys. Rev. D 93, 034035 (2016).

[5] D. Ebert, R. N. Faustov, and V. O. Galkin, Eur. Phys. J. C 66, 197 (2010).

[6] R. N. Cahn and J. D. Jackson, Phys. Rev. D 68, 037502 (2003).

[7] T. Barnes, F. E. Close, and H. J. Lipkin, Phys. Rev. D 68, 054006 (2003).

[8] A. P. Szczepaniak, Phys. Lett. B 567, 23 (2003).

[9] Y.-Q. Chen and X.-Q. Li, Phys. Rev. Lett. 93, 232001 (2004).

[10] F.-K. Guo, P.-N. Shen, H.-C. Chiang, R.-G. Ping, and B.-S. Zou, Phys. Lett. B 641, 278 (2006).

[11] F.-K. Guo, P.-N. Shen, and H.-C. Chiang, Phys. Lett. B 647, 133 (2007).

[12] H.-Y. Cheng and W.-S. Hou, Phys. Lett. B 566, 193 (2003).
[13] L. Maiani, F. Piccinini, A. D. Polosa, and V. Riquer, Phys. Rev. D 71, 014028 (2005).

[14] T. E. Browder, S. Pakvasa, and A. A. Petrov, Phys. Lett. B 578, 365 (2004).

[15] E. van Beveren and G. Rupp, Phys. Rev. Lett. 91, 012003 (2003).

[16] K. Abe et al. (Belle Collaboration), Phys. Rev. D 69, 112002 (2004).

[17] J. M. Link et al. (FOCUS Collaboration), Phys. Lett. B 586, 11 (2004).

[18] T. Mehen and R. P. Springer, Phys. Rev. D 72, 034006 (2005).

[19] B. Ananthanarayan, S. Banerjee, K. Shivaraj, and A. Upadhyay, Phys. Lett. B 651, 124 (2007).

[20] P. Colangelo, F. De Fazio, F. Giannuzzi, and S. Nicotri, Phys. Rev. D 86, 054024 (2012).

[21] M. Tanabashi et al. (Particle Data Group), Phys. Rev. D 98, 030001 (2018).

[22] B. Aubert et al. (BABAR Collaboration), Phys. Rev. D 79, 112004 (2009). 
[23] R. Aaij et al. (LHCb Collaboration), Phys. Rev. D 92, 012012 (2015).

[24] R. Aaij et al. (LHCb Collaboration), Phys. Rev. D 92 , 032002 (2015).

[25] E. E. Kolomeitsev and M. F. M. Lutz, Phys. Lett. B 582, 39 (2004).

[26] J. Hofmann and M. F. M. Lutz, Nucl. Phys. A733, 142 (2004).

[27] D. Gamermann, E. Oset, D. Strottman, and M. J. Vicente Vacas, Phys. Rev. D 76, 074016 (2007).

[28] F.-K. Guo, C. Hanhart, S. Krewald, and U.-G. Meißner, Phys. Lett. B 666, 251 (2008).

[29] F.-K. Guo, C. Hanhart, and U.-G. Meißner, Eur. Phys. J. A 40, 171 (2009).

[30] L. Liu, K. Orginos, F.-K. Guo, C. Hanhart, and U.-G. Meißner, Phys. Rev. D 87, 014508 (2013).

[31] D.-L. Yao, M.-L. Du, F.-K. Guo, and U.-G. Meißner, J. High Energy Phys. 11 (2015) 058.

[32] M.-L. Du, F.-K. Guo, U.-G. Meißner, and D.-L. Yao, Eur. Phys. J. C 77, 728 (2017).

[33] X.-Y. Guo, Y. Heo, and M. F. M. Lutz, Phys. Rev. D 98, 014510 (2018).

[34] Z.-H. Guo, L. Liu, U.-G. Meißner, J. A. Oller, and A. Rusetsky, Eur. Phys. J. C 79, 13 (2019).

[35] M.-L. Du, M. Albaladejo, P. Fernandez-Soler, F.-K. Guo, C. Hanhart, U.-G. Meißner, J. Nieves, and D.-L. Yao, Phys. Rev. D 98, 094018 (2018).

[36] F.-K. Guo, C. Hanhart, U.-G. Meißner, Q. Wang, Q. Zhao, and B.-S. Zou, Rev. Mod. Phys. 90, 015004 (2018).

[37] M. Albaladejo, P. Fernandez-Soler, F.-K. Guo, and J. Nieves, Phys. Lett. B 767, 465 (2017).

[38] R. Aaij et al. (LHCb Collaboration), Phys. Rev. D 94, 072001 (2016).

[39] M. J. Savage and M. B. Wise, Phys. Rev. D 39, 3346 (1989); 40, 3127(E) (1989).

[40] M. Albaladejo, P. Fernandez-Soler, J. Nieves, and P. G. Ortega, Eur. Phys. J. C 78, 722 (2018).

[41] G. Moir, M. Peardon, S. M. Ryan, C. E. Thomas, and D. J. Wilson, J. High Energy Phys. 10 (2016) 011.

[42] G. S. Bali, S. Collins, A. Cox, and A. Schäfer, Phys. Rev. D 96, 074501 (2017).
[43] A. Dobado and J. R. Peláez, Phys. Rev. D 56, 3057 (1997).

[44] J. A. Oller and E. Oset, Nucl. Phys. A620, 438 (1997); A652, 407 (1999).

[45] J. A. Oller, E. Oset, and J. R. Peláez, Phys. Rev. D 59, 074001 (1999); 60, 099906(E) (1999); 75, 099903(E) (2007).

[46] J. A. Oller and U.-G. Meißner, Phys. Lett. B 500, 263 (2001).

[47] A. Gomez Nicola and J. R. Peláez, Phys. Rev. D 65, 054009 (2002).

[48] S. R. Coleman, J. Wess, and B. Zumino, Phys. Rev. 177, 2239 (1969).

[49] C. G. Callan, Jr., S. R. Coleman, J. Wess, and B. Zumino, Phys. Rev. 177, 2247 (1969).

[50] J. Gasser and H. Leutwyler, Ann. Phys. (N.Y.) 158, 142 (1984).

[51] J. Gasser and H. Leutwyler, Nucl. Phys. B250, 465 (1985).

[52] S. Weinberg, Physica (Amsterdam) 96A, 327 (1979).

[53] M.-L. Du, F.-K. Guo, and U.-G. Meißner, J. High Energy Phys. 10 (2016) 122.

[54] M.-L. Du, F.-K. Guo, and U.-G. Meißner, J. Phys. G 44, 014001 (2017).

[55] J. Bijnens and A. Celis, Phys. Lett. B 680, 466 (2009).

[56] R. Aaij et al. (LHCb Collaboration), Phys. Rev. D 90, 072003 (2014).

[57] R. Aaij et al. (LHCb Collaboration), Phys. Rev. D 91, 092002 (2015); 93, 119901(E) (2016).

[58] E. Oset et al., Int. J. Mod. Phys. E 25, 1630001 (2016).

[59] M. Albaladejo, D. Jido, J. Nieves, and E. Oset, Eur. Phys. J. C 76, 300 (2016).

[60] C. Zemach, Phys. Rev. 133, B1201 (1964).

[61] B. Aubert et al. (BABAR Collaboration), Phys. Rev. Lett. 100, 171803 (2008).

[62] J. Wiechczynski et al. (Belle Collaboration), Phys. Rev. D 80, 052005 (2009).

[63] J. Wiechczynski et al. (Belle Collaboration), Phys. Rev. D 91, 032008 (2015).

[64] E. Kou et al. (Belle II Collaboration), arXiv:1808.10567.

[65] A. Cerri et al., arXiv:1812.07638. 